\title{
Modular isogeny complexes
}

\author{
CHARLES REZK
}

\begin{abstract}
We describe a vanishing result on the cohomology of a cochain complex associated to the moduli of chains of finite subgroup schemes on elliptic curves. These results have applications to algebraic topology, in particular to the study of power operations for Morava $E$-theory at height 2.
\end{abstract}

$11 \mathrm{G} 18 ; 55 \mathrm{~N} 34,55 \mathrm{~S} 25,11 \mathrm{G} 15$

\section{Introduction}

Let $A$ be an abelian group (possibly infinite), $k$ a commutative ring, $p$ a prime and $r \geq 1$. Let $\mathscr{K}_{p^{r}}^{\bullet}(A ; k)$ be the cochain complex defined by

$\mathscr{K}_{p^{r}}^{q}(A ; k)=\prod_{G_{1}, \ldots, G_{q}} k, \quad(\delta f)\left(G_{1}, \ldots, G_{q+1}\right)=\sum(-1)^{k} f\left(G_{1}, \ldots, \widehat{G_{k}}, \ldots, G_{q+1}\right)$,

where the product is taken over the set of all increasing chains $G_{1} \subsetneq \cdots \subsetneq G_{q}$ of subgroups of $A$ such that the largest subgroup in the chain $G_{q}$ has order $p^{r}$. One can show that

(1) $H^{q} \mathscr{Y}_{p^{r}}^{\bullet}(A ; k)=0$ unless $q=r$,

(2) $H^{r} \mathscr{K}_{p^{r}}(A ; k)$ is a free $k$-module, of rank $n p^{r(r-1) / 2}$, where $n$ is the number of distinct subgroups of $A$ which are isomorphic to $(\mathbb{Z} / p)^{r}$.

This is not a very deep result; it is essentially the theorem of Solomon and Tits (see Solomon [10]) on the cohomology of the Tits building of $\mathrm{GL}_{r}(\mathbb{Z} / p)$. (This is described in Section 2.)

Nonetheless, we may consider an elliptic curve $E$ over an algebraically closed field $k$, of characteristic 0 or finite characteristic other than $p$. Taking $A=E(k)$ the group of $k$-rational points on $E$, we see that since the $p$-torsion in $A$ is isomorphic to $\left(\mathbb{Q}_{p} / \mathbb{Z}_{p}\right)^{2}$, we must have $H^{r} \mathscr{K}_{p^{r}}(E(k) ; k)=0$ for $r \geq 3$, while $H^{1} \mathscr{K}_{p}(E(k) ; k)$ and $H^{2} \varkappa_{p}(E(k) ; k)$ are free modules of ranks $p+1$ and $p$ respectively. 


\subsection{Subgroups of elliptic curves as a moduli problem}

Let (Ell) denote the category whose objects $E / S$ are elliptic schemes $E$ over a base scheme $S$, and whose morphisms $E / S \rightarrow E^{\prime} / S^{\prime}$ are fiber squares.

Given an elliptic curve $E / S$, let $[N-\operatorname{Isog}](E / S)$ denote the set of locally free finite commutative $S$-subgroup schemes $G \subset E$ which are rank $N$ over $S$; see Katz and Mazur [4, Section 6.5]. According to [4, 6.5.1], $[N-\mathrm{Isog}]$ is relatively representable and finite over (Ell). That is, given an elliptic curve $E / S$, the functor on $(\mathrm{Sch} / S)$ given by $T \mapsto[N-\operatorname{Isog}]\left(E_{T} / T\right)$ is represented by an $S$-scheme $[N-\operatorname{Isog}]_{E / S}[4$, Section 4.2], which is finite and flat, and hence locally free, over $S$; furthermore, the rank of $[N-\operatorname{Isog}]_{E / S}$ is constant and is equal to the number of subgroups of order $N$ in $(\mathbb{Q} / \mathbb{Z})^{2}$.

For each element $G \subset E$ of $[N-\operatorname{Isog}](E / S)$ there is an $N$-isogeny $f: E \rightarrow E^{\prime}$ of curves over $S$ with kernel $G$, unique up to unique isomorphism in the category of isogenies with domain $E$, hence the notation.

Now suppose that $S=\operatorname{Spec} A$ is an affine scheme. Then $[N-\operatorname{Isog}](E / S)$ is necessarily affine. I write $\mathscr{Y}_{N}(E / S)$ for the function ring of $[N-\operatorname{Isog}](E / S)$; it is naturally an $A$-algebra, finite and locally free. Furthermore, given a map $T \rightarrow S$ of schemes induced by a map $A \rightarrow B$ of rings, we have $\mathscr{Y}_{N}\left(E_{T} / T\right) \approx \mathscr{S}_{N}(E / S) \otimes_{A} B$.

Remark 1.2 If we use the language of moduli stacks, then we can say that $\mathscr{Y}_{N}$ is a coherent sheaf on the moduli stack of elliptic curves; in fact, it is the direct image of the structure sheaf along the evident map of stacks $M_{[N-\mathrm{Isog}]} \rightarrow M_{\text {(Ell) }}$ which forgets about the subgroup. Likewise, the complex $\mathcal{K}_{N}^{\bullet}$ to be defined below is a complex of coherent sheaves on $M_{(\text {Ell) }}$. We prefer to avoid stack language and discuss these objects in more concrete terms.

\subsection{Chains of subgroups}

For integers $N_{1}, \ldots, N_{q} \geq 1$, let $\left[N_{1}, \ldots, N_{q}-\operatorname{Isog}\right](E / S)$ denote the set of sequences $\underline{G}=\left(G_{1} \subsetneq \cdots \subsetneq G_{q}\right)$, where $G_{i}$ is a locally free commutative $S$-subgroup scheme of $E$ of rank $N_{1} \cdots N_{i}$, and where $G_{i-1}$ is a subscheme of $G_{i}$. Thus, $G_{i} / G_{i-1}$ is a finite group scheme over $S$ of rank $N_{i}$. As above, given $E / S$, the functor on $(\mathrm{Sch} / S)$ given by $T \mapsto\left[N_{1}, \ldots, N_{q}-\operatorname{Isog}\right]\left(E_{T} / T\right)$ is represented by an $S$-scheme $\left[N_{1}, \ldots, N_{q}-\operatorname{Isog}\right]_{E / S}$, finite and locally free over $S$. Again, if $S=\operatorname{Spec}(A)$ is affine, so is $\left[N_{1}, \ldots, N_{q}-\mathrm{Isog}\right]_{E / S}$ with function ring denoted $\mathscr{S}_{N_{1}, \ldots, N_{q}}(E / S)$, and this ring is finite and locally free as an $A$ module. 
As usual, elements $\underline{G}$ of $\left[N_{1}, \ldots, N_{q}-\operatorname{Isog}\right](E / S)$ can be identified with suitable isomorphism classes of sequences

$$
E \stackrel{f_{1}}{\longrightarrow} E_{1} \stackrel{f_{2}}{\longrightarrow} \cdots \stackrel{f_{q}}{\longrightarrow} E_{q}
$$

of isogenies with $\operatorname{Ker}\left(f_{i} f_{i-1} \cdots f_{i}\right)=G_{i}$.

There are maps $U_{i}:\left[N_{1}, \ldots, N_{q}-\right.$ Isog $] \rightarrow\left[N_{1}, \ldots, N_{i-1} N_{i}, \ldots, N_{q}-\right.$ Isog $]$ for $i=$ $1, \ldots, q$, defined by forgetting the $i$-th group in the sequence $\left(G_{1} \subsetneq \ldots \subsetneq G_{q}\right)$. We write $u_{i}: \mathscr{Y}_{N_{1}, \ldots, N_{i-1} N_{i}, \ldots, N_{q}}(E / S) \rightarrow \mathscr{Y}_{N_{1}, \ldots, N_{q}}(E / S)$ for the corresponding map of rings, when $S$ is affine.

There are also evident maps $\left[N_{1}, \ldots, N_{q}-\right.$ Isog $] \rightarrow[1-$ Isog $]$, which forget the finite subgroups. I'll write $s: \mathscr{S}_{1}(E / S) \rightarrow \mathscr{I}_{N_{1}, \ldots, N_{q}}(E / S)$ for the corresponding map of rings, which is precisely the map which exhibits $\mathscr{S}_{N_{1}, \ldots, N_{q}}(E / S)$ as an $A$-algebra.

\subsection{The modular $N$-isogeny complex}

Fix an elliptic curve $E / S$, where $S=\operatorname{Spec}(A)$ is an affine scheme, and let $N \geq 1$. We define a bounded cochain complex $\mathscr{K}_{N}^{\bullet}(E / S)$ of $A$-modules as follows. Set

$$
\mathscr{K}_{N}^{q}(E / S)=\prod_{N_{1}, \ldots, N_{q}} \mathscr{S}_{N_{1}, \ldots, N_{q}}(E / S)
$$

where the product runs through all tuples $\left(N_{1}, \ldots, N_{q}\right)$ of integers of length $q$ such that $N_{1} \cdots N_{q}=N$ and each $N_{i}>1$. If $q=0$ and $N>1$, we have $\mathscr{K}_{N}^{0}=0$. We also stipulate that if $N=1$, then $\mathscr{K}_{1}^{0}=\mathscr{Y}_{1}(E / S)=A$ and $\mathscr{K}_{1}^{q}=0$ for $q>0$. Given an element $f \in \mathscr{K}_{N}^{q}(E / S)$, write $f_{N_{1}, \ldots, N_{q}}$ for its component in $\mathscr{S}_{N_{1}, \ldots, N_{q}}(E / S)$. We define the coboundary map $\delta: \mathscr{K}_{N}^{q-1} \rightarrow \mathscr{K}_{N}^{q}$ by the formula

$$
(\delta f)_{N_{1}, \ldots, N_{q}}=\sum_{i=1}^{q-1}(-1)^{i} u_{i}\left(f_{N_{1}, \ldots, N_{i} N_{i+1}, \ldots, N_{q}}\right),
$$

where $u_{i}$ is as defined in Section 1.3.

We will call $\mathcal{K}_{N}^{\bullet}$ the modular $N$-isogeny complex, for lack of a better name. 
We are mainly interested in the case when $N=p^{r}$ for some prime $p$. For small values of $r$ these complexes appear as follows.

$$
\begin{aligned}
\mathscr{K}_{1}^{\bullet}: & \mathscr{S}_{1} \\
\mathscr{K}_{p}^{\bullet}: & 0 \longrightarrow \mathscr{S}_{p} \\
\mathscr{K}_{p^{2}}^{\bullet}: & 0 \longrightarrow \mathscr{S}_{p^{2}} \stackrel{u_{1}}{\longrightarrow} \mathscr{S}_{p, p} \\
\mathscr{K}_{p^{3}}^{\bullet}: & 0 \longrightarrow \mathscr{S}_{p^{3}} \stackrel{\left(u_{1}, u_{1}\right)}{\longrightarrow} \mathscr{I}_{p^{2}, p} \times \mathscr{Y}_{p, p^{2}} \stackrel{\left(u_{1},-u_{2}\right)}{\longrightarrow} \mathscr{S}_{p, p, p}
\end{aligned}
$$

\subsection{Main theorem}

Our main result is the following.

Theorem 1.6 Let $E / S$ be an elliptic curve over an affine scheme $S=\operatorname{Spec} A$, and let $p$ be a prime.

(1) If $j \neq r$, then $H^{j} \mathscr{K}_{p^{r}}^{\bullet}(E / S)=0$.

(2) $H^{r} \mathcal{K}_{p^{r}}^{\bullet}(E / S)$ is finite and locally free as an $A$-module.

(3) $H^{r} K_{p^{r}}^{\bullet}(E / S)=0$ if $r \geq 3$.

(4) There are natural isomorphisms of $A$-modules

$$
\begin{gathered}
H^{0} \mathscr{K}_{1}^{\bullet}(E / S)=\mathscr{Y}_{1}(E / S), \quad H^{1} \mathscr{K}_{p}^{\bullet}(E / S)=\mathscr{Y}_{p}(E / S), \\
H^{2} \mathscr{K}_{p^{2}}^{\bullet}(E / S) \approx \operatorname{Cok}\left[s: \mathscr{Y}_{1}(E / S) \rightarrow \mathscr{S}_{p}(E / S)\right] .
\end{gathered}
$$

In particular, $H^{0} \mathscr{K}_{1}^{\bullet}(E / S), H^{1} \mathscr{K}_{p}^{\bullet}(E / S)$ and $H^{2} \mathscr{K}_{p^{2}}(E / S)$ are locally free over $A$ of ranks $1, p+1$, and $p$ respectively.

\subsection{Proofs of (3) and (4)}

The main claims of the theorem are (1) and (2), and we can deduce the remaining statements from these.

Proof of (3) using (1) and (2) We use a "dimension count", meaning we compare ranks of finite and locally free $A$-modules. Write $\mathscr{Y}_{N_{1}, \ldots, N_{q}}$ for $\mathscr{Y}_{N_{1}, \ldots, N_{q}}(E / S)$, and $\mathscr{K}_{N_{1}, \ldots, N_{q}}^{\bullet}$ for $\mathscr{Y}_{N_{1}, \ldots, N_{q}}^{\bullet}(E / S)$, with $S=\operatorname{Spec} A$. We have that $\mathscr{S}_{N}$ is locally free of constant rank as an $A$-module, and that this rank is equal to the number of subgroups of order $N$ in $(\mathbb{Q} / \mathbb{Z})^{2}$. Thus, if we write $\sigma(N)=\sum_{d \mid N} d$ for the number of such subgroups, then we have $\operatorname{rank} \mathscr{S}_{N}=\sigma(N)$, and more generally,

$$
\operatorname{rank} \mathscr{S}_{N_{1}, \ldots, N_{r}}=\sigma\left(N_{1}\right) \cdots \sigma\left(N_{r}\right)
$$


Counting such subgroups of $p$-th power order leads to a generating function

$$
f(T)=\sum_{r \geq 0} \operatorname{rank} \mathscr{S}_{p^{r}} T^{r}=\sum_{r \geq 0} \sigma\left(p^{r}\right) T^{r}=[(1-T)(1-p T)]^{-1},
$$

and from this we obtain

$$
\begin{aligned}
\sum_{r \geq 0} \operatorname{rank} \mathscr{K}_{p^{r}}^{q} T^{r} & =\sum_{r_{1}, \ldots, r_{q}>0} \operatorname{rank} \mathscr{S}_{p^{r_{1}, \ldots, p^{r q}}} T^{r_{1}+\cdots+r_{q}} \\
& =\sum_{r_{1}, \ldots, r_{q}>0} \sigma\left(r_{1}\right) \cdots \sigma\left(r_{q}\right) T^{r_{1}+\cdots+r_{q}}=(f(T)-1)^{q}
\end{aligned}
$$

By (2), the cohomologies $H^{j} \mathscr{K}_{p^{r}}$ are finite and locally free over $A$, so

$$
\sum_{j}(-1)^{j} \operatorname{rank} H^{j} \mathscr{K}_{p^{r}}=\sum_{j}(-1)^{j} \operatorname{rank} \mathscr{K}_{p^{r}}^{j}
$$

Hence by (1) we have

$$
\sum_{r \geq 0} \operatorname{rank} H^{r} \mathscr{K}_{p^{r}}^{\bullet}(-T)^{r}=\sum_{q \geq 0}(1-f(T))^{q}=(f(T))^{-1}=(1-T)(1-p T) .
$$

Thus, $H^{r} \mathscr{K}_{p^{r}}^{\bullet}=0$ if $r \geq 3$.

Proof of (4) using (1) and (2) The only nontrivial statement is that for $H^{2} \mathscr{K}_{p^{2}}$. Consider the following commutative square of moduli problems:

$$
\begin{aligned}
& {[p-\mathrm{I} \operatorname{sog}] \longrightarrow\left[\stackrel{f}{\rightarrow} E^{\prime}\right) \mapsto(E) \longrightarrow[1-\mathrm{Isog}]} \\
& \left(E \stackrel{f}{\rightarrow} E^{\prime}\right) \mapsto\left(E \stackrel{f}{\rightarrow} E^{\prime} \stackrel{\widehat{f}}{\rightarrow} E\right) \downarrow \quad \downarrow(E) \mapsto(E \stackrel{[p]}{\longrightarrow} E) \\
& {[p, p-\mathrm{Isog}] \underset{\left(E \stackrel{f}{\rightarrow} E^{\prime} \stackrel{g}{\rightarrow} E^{\prime \prime}\right) \mapsto\left(E \stackrel{g f}{\longrightarrow} E^{\prime \prime}\right)}{\longrightarrow}\left[p^{2}-\mathrm{Isog}\right]}
\end{aligned}
$$

The commutativity of this square encodes the identity $\hat{f} f=[p]$, where $\hat{f}$ is the dual isogeny to a $p$-isogeny $f$. This square gives a commutative square of $A$-algebras:

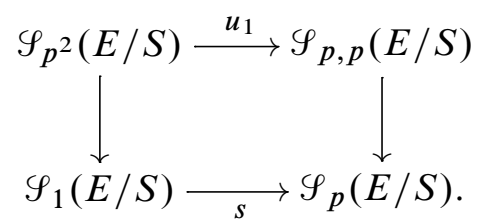

The map $s: \mathscr{Y}_{1} \rightarrow \mathscr{Y}_{p}$ is injective with locally free cokernel (it's faithfully flat). The rank of the cokernel is constant over $A$ with $\operatorname{rank}_{A} \mathscr{S}_{p}(E / S) / \mathscr{I}_{1}(E / S)=(p+1)-1=p$. 
Using statement (2) of Theorem 1.6, $\mathscr{I}_{p, p}(E / S) / \mathscr{I}_{p^{2}}(E / S) \approx H_{2} \mathscr{K}_{p^{2}}(E / S)$ is locally free of rank $p$ as well. The vertical maps in the square are epimorphisms since they admit sections, so the induced map $\mathscr{S}_{p, p} / \mathscr{S}_{p^{2}} \rightarrow \mathscr{Y}_{p} / \mathscr{S}_{1}$ is an epimorphism between locally free $A$-modules of the same rank, hence is an isomorphism.

\subsection{Proof of (1) and (2)}

To complete the proof Theorem 1.6, note that if $E / S$ is an elliptic curve, then $S$ admits a cover by open affines $U_{i}$ such that $E_{U_{i}} / U_{i}$ is given by a Weierstrass equation, and so is the pullback of an elliptic curve over a scheme of finite type. Thus, we may assume without loss of generality that $S$ is of finite type, and in this case we can replace "locally free" with "projective" in the statement of the theorem.

We use the following application of Nakayama's lemma.

Proposition 1.9 Let $A$ be a commutative ring, and let

$$
P^{\bullet}=\left(0 \rightarrow P^{0} \rightarrow \cdots \rightarrow P^{n} \rightarrow 0\right)
$$

be a bounded cochain complex of finitely generated projective $A$-modules. The following are equivalent.

(1) $H^{j}\left(P^{\bullet}\right)=0$ for $j \neq n$, and $H^{n}\left(P^{\bullet}\right)$ is a finitely generated projective $A$ module.

(2) For every ring homomorphism $A \rightarrow B$, we have $H^{j}\left(P^{\bullet} \otimes_{A} B\right)=0$ for $j \neq n$, and $H^{n}\left(P^{\bullet} \otimes_{A} B\right)$ is a finitely generated projective $B$-module.

(3) For every maximal ideal $\mathfrak{m}$ of $A$, we have $H^{j}\left(P^{\bullet} \otimes_{A} A / \mathfrak{m}\right)=0$ for $j \neq n$.

Proof Condition (1) implies that $P^{\bullet}$ is chain homotopy equivalent to the complex consisting of $H^{n}\left(P^{\bullet}\right)$ in degree $n$, and thus (2) follows. Clearly (2) implies (3).

To show that (3) implies (1), first consider the special case where $A$ is a local ring (in which case the $P^{j}$ are finitely generated free modules), and write $k=A / \mathfrak{m}$. This case is easily proved by induction on $n$, the case of $n=0$ being immediate. If $n>0$, we have $H^{0}\left(P^{\bullet} \otimes_{A} k\right)=0$, so that $P^{0} \otimes_{A} k \rightarrow P^{1} \otimes_{A} k$ is injective. Choose a free $A$-module $Q$ and a map $Q \rightarrow P^{1}$ so that the induced map $g: P^{0} \oplus Q \rightarrow P^{1}$ induces an isomorphism after tensoring down to $k$. Thus $g$ is itself an isomorphism by Nakayama's lemma, and we conclude that $P^{\bullet}$ is chain-homotopy-equivalent to the complex obtained from $P^{\bullet}$ by replacing $P^{0}$ with 0 and $P^{1}$ with $Q$. The induction hypothesis applies to $Q$, so the claim is proved. 
For general $A$, note that $H^{*}\left(P^{\bullet}\right) \otimes_{A} A_{\mathfrak{m}} \approx H^{*}\left(P^{\bullet} \otimes_{A} A_{\mathfrak{m}}\right)$, and so from what we have shown it follows that $H^{j}\left(P^{\bullet}\right)=0$ for $j \neq n$. The exact sequence $P^{n-1} \rightarrow$ $P^{n} \rightarrow H^{n}\left(P^{\bullet}\right) \rightarrow 0$ shows that $H^{n}\left(P^{\bullet}\right)$ is finitely presented, and therefore projective since it is locally free.

Thus, the proof of Theorem 1.6 reduces to showing statements (1) and (2) of Theorem 1.6 for elliptic curves $E / S$, where $S=\operatorname{Spec} k$ for a field $k$. There are three cases we must consider, for a given prime $p$.

(A) The field $k$ has characteristic not equal to $p$.

(B) The field $k$ has characteristic $p$, and $E$ is an ordinary elliptic curve.

(C) The field $k$ has characteristic $p$, and $E$ is a supersingular curve.

In each case we may without loss of generality assume that $k$ algebraically closed.

There is a trick (inspired by its use in [4, Chapter 5]) which allows us to deduce case (B) from case (C).

(1) Note that for $E / S$, the question of whether the claims of Theorem 1.6 are true for $E / S$ depends only on the underlying $p$-divisible group of $E$. Over $S=\operatorname{Spec}(k)$ with $k$ algebraically closed, there are only three $p$-divisible groups which can appear, according to the three cases: (A) $\left(\mathbb{Q}_{p} / \mathbb{Z}_{p}\right)^{2}$, (B) $\widehat{\mathbb{G}}_{m} \times \mathbb{Q}_{p} / \mathbb{Z}_{p}$, and (C) the unique formal group of height 2 over $k$.

In particular, to prove the theorem for case (B), it suffices to prove it for one ordinary curve.

(2) Given $E / S$ and $k \geq r$, let $U$ be the set of points $s$ in $S$ for which we have that $H^{j} \mathcal{K}_{p^{r}}^{\bullet}(E \otimes k(s) / \operatorname{Spec} k(s))=0$ for $j \neq r$. If $S$ is of finite type over $\operatorname{Spec}(\mathbb{Z})$, then $U$ is a Zariski open subset of $S$.

In the moduli stack of elliptic curves, every open neighborhood of a supersingular point contains an ordinary point; more precisely, the moduli stack admits an étale cover by a collection $\left\{E_{i} / S_{i}\right\}$ where each $S_{i}$ is finite type over $\operatorname{Spec}(\mathbb{Z})$, and such that each open neighborhood of a supersingular point in $S_{i}$ contains an ordinary point. Therefore (C) implies (B).

We'll prove cases (A) and (C) by direct calculation. Case (A) has an easy combinatorial proof, while the proof of case (C) amounts to an explicit calculation of the complex $\mathcal{K}_{p^{r}}^{\bullet}$ at the universal deformation of a supersingular curve, and will constitute the main part of the paper.

Thus, in Section 2 we prove case (A) (Propositions 2.4 and 2.5). In Section 3 we give an explicit description Proposition 3.18 of the complexes $\mathscr{K}_{p^{r}}^{\bullet}(E / S)$, where $E / S$ is 
the universal deformation of a suitable supersingular curve; the main points we need are contained in [4, Chapter 13]. Finally, in Section 4 we use this explicit description to prove Theorem 1.6 for the universal deformation (Propositions 4.2 and 4.20), from which case (C) follows directly; this part of the argument is essentially an application of the proof of the "PBW basis theorem" of Priddy [6].

It is possible to prove (B) by an explicit calculation such as that for (C) given here, and I hope to give that calculation elsewhere.

\subsection{Applications to algebraic topology}

This work was motivated by applications to elliptic cohomology (some actual, some conjectural). A detailed discussion is outside the scope of this paper; however, we can briefly describe a couple of points of contact.

To every one-dimensional formal group $G_{0}$ of finite height over a perfect field $k$ of characteristic $p$, there is an associated generalized cohomology theory $E=E_{G_{0} / k}$, called the Morava $E$-theory associated to $G_{0} / k$. The theory $E$ is 2 -periodic and complex orientable, and its formal group $G / \pi_{0} E$ is the universal deformation of $G_{0} / k$ in the sense of Lubin and Tate. In particular, $\pi_{0} E \approx \mathbb{W} k \llbracket x_{1}, \ldots, x_{n-1} \rrbracket$, where $n$ is the height of $G_{0}$.

To each cohomology theory $E$ is associated a certain ring $\mathcal{P}$ of operations, called the ring of power operations; the ring $\mathcal{P}$ contains the coefficient ring $\pi_{0} E$, but not centrally. By work of Strickland [11; 12] (see Rezk [9] for an exposition), the ring $\mathcal{P}$ encodes in a precise way all information about the moduli of finite subgroups of the formal group $G$.

The reduction of $\mathcal{P}$ modulo $p$ makes a crucial appearance in this paper. More precisely, suppose that $G_{0} / \mathbb{F}_{p^{2}}$ is the formal completion of a standard supersingular curve (defined in Section 3.8). Its universal deformation lives over the ring $\mathbb{W} \mathbb{F}_{p^{2}} \llbracket x \rrbracket$; let $\mathcal{P}$ be the ring of power operations for the associated Morava $E$-theory. Then, as a result of the calculations of Section 3 and Section 4, we have an isomorphism of rings

$$
\mathcal{P} \otimes \mathbb{Z} / p \approx \Gamma,
$$

where $\Gamma$ is the ring described in terms of explicit generators and relations in Section 4; see Section 4.8 and Section 4.12, especially (4.9), (4.10) and (4.11). We note that the resulting admissible monomial basis we give for $\Gamma$ is compatible with the calculations of Kashiwabara [3], though in his context it is not possible to give an algebra structure.

In the 1990s, Matt Ando, Mike Hopkins and Neil Strickland conjectured that for any Morava $E$-theory associated to any formal group, its ring $\mathcal{P}$ of power operations would 
be what is called a Koszul ring. I have proved this conjecture (see [8], forthcoming), using methods of algebraic topology. The argument of Section 4 of this paper gives an independent proof for the case of Morava $E$-theory of height 2 formal groups, in some ways along the lines that Ando, Hopkins and Strickland envisioned.

The complexes $\mathscr{K}_{\ell^{r}}^{\bullet}(E / S)$ when $\ell$ is invertible over the scheme $S$ are closely related to work (see Behrens and Lawson [2]) on approximations to the $K(2)$-local sphere at a prime $p \neq \ell$, which are constructed using $\ell-$ th power isogenies on a supersingular curve at $p$.

Acknowledgments I'd like to thank Kevin Buzzard, who directed my attention to the appropriate sections in Katz and Mazur [4] and helpfully criticized my fumbling formulation of an early version of some of this. I would also like to thank Nick Kuhn for supplying the observation that led to the formulation of part (4) of the main theorem.

The author was supported under NSF grant DMS-1006054.

\section{The proof of the theorem over fields with $p$ invertible}

Let $k$ be an algebraically closed field in which $p$ is invertible, let $S=\operatorname{Spec} k$, and let $E / S$ be an elliptic curve. In this section we show that statements (1) and (2) of Theorem 1.6 are true for such a curve.

In this case, we have that $E\left[p^{\infty}\right] \approx\left(\mathbb{Q}_{p} / \mathbb{Z}_{p}\right)^{2}$, and thus that finite subgroup schemes $G \subset E$ of $p$-th power order correspond to finite subgroups of $\left(\mathbb{Q}_{p} / \mathbb{Z}_{p}\right)^{2}$. The complex $\mathscr{K}_{p^{r}}(E / S)$ thus admits a combinatorial description, which we now give.

\subsection{The order complex of subgroups of an abelian group}

Let $G$ be an abelian group, and let $P_{G}$ denote the poset of proper nontrivial subgroups of $G$. This poset is associated to an abstract simplicial complex, called its order complex, which we also denote $P_{G}$. This is an abstract simplicial complex whose vertices correspond to proper nontrivial subgroups of $G$, and whose $q$-simplices correspond to chains $\left[0 \subsetneq G_{1} \subsetneq \cdots \subsetneq G_{q} \subsetneq G\right]$ of subgroups $G_{i}$ of $G$.

Note that $P_{\mathbb{Z} / p}$ and $P_{0}$ are empty.

Given a simplicial complex $X$ with some chosen ordering of its vertices, let $C_{\bullet}(X)$ denote the usual chain complex associated to $X$ with integer coefficients, and let $\widetilde{C}_{\bullet}(X)$ denote the mapping fiber of the augmentation $C_{\bullet}(X) \rightarrow \mathbb{Z}$. Thus $\widetilde{C}_{q}(X)$ is free abelian group on the $q$-simplices of $X$ if $q \geq 0$, and $\widetilde{C}_{-1}(X)=\mathbb{Z}$. 
Proposition 2.2 Let $G$ be a finite abelian $p$-group, with $G \neq 0$.

(1) If $p G \neq 0$, then $H_{q}\left(\widetilde{C}_{\bullet}\left(P_{G}\right)\right)=0$ for all $q$.

(2) If $p G=0$, so that $G \approx(\mathbb{Z} / p)^{\times r}$, then $H_{q}\left(\widetilde{C}_{\bullet}\left(P_{G}\right)\right)=0$ for $q \neq r-2$, while $H_{r-2} \widetilde{C}_{\bullet}\left(P_{G}\right)$ is a free abelian group.

Proof In case (1), there exists a proper subgroup $V \subsetneq G$ which is cyclic of order $p$ and is not a summand of $G$. Thus, given any proper nontrivial subgroup $H$ of $G$, the subgroup $H+V$ is again proper and nontrivial. The chain of inclusions $H \subseteq H+V \supseteq V$ defines a pair of homotopies between self-maps of the geometric realization $\left|P_{G}\right|$, which relate the identity map of $\left|P_{G}\right|$ to a constant map. Thus, $\left|P_{G}\right|$ is contractible, and the result on homology follows.

Case (2) is a special case of the theorem of Solomon and Tits [10], which says that $\left|P_{G}\right|$ is homotopy equivalent to a wedge (one-point union) of $(r-2)$-dimensional spheres if $r \geq 2$. An elegant proof which applies in this particular case is given by Quillen [7, Section 2].

In this paper, we actually only need part (2) of Proposition 2.2 in the cases of $r=1$ and 2, where it is trivial since $P_{(\mathbb{Z} / p)^{r}}$ is empty or 0 -dimensional in these cases.

\subsection{Description of $\mathcal{K}_{p^{r}}^{\bullet}$}

Now we define for each finite abelian $p$-group $G$ and each abelian group $M$ a cochain complex $D_{G}^{\bullet}(M)$ as follows. If $G \approx 0$, we set $D_{G}^{0}(M) \approx M$ and $D_{G}^{q}(M)=0$ for $q \neq 0$. If $G \not \approx 0$, we set

$$
D_{G}^{q}(M)=\operatorname{Hom}\left(\tilde{C}_{q-2}\left(P_{G}\right), M\right),
$$

and the coboundary map of $D_{G}^{\bullet}(M)$ be induced by the boundary map of $\widetilde{C}_{\bullet-2}\left(P_{G}\right)$. We have the following immediate consequence of Proposition 2.2.

Proposition 2.4 Let $G$ be a finite abelian $p$-group.

(1) If $p G \neq 0$, then $H^{q}\left(D_{G}^{\bullet}(M)\right)=0$ for all $q$.

(2) If $p G=0$, so that $G \approx(\mathbb{Z} / p)^{r}$, then $H_{q}\left(D_{G}^{\bullet}(M)\right)=0$ for $q \neq r$.

Now we consider our elliptic curve $E$ over an algebraically closed field $k$ in which $p$ is invertible. 
Proposition 2.5 Let $E / \operatorname{Spec}(k)$ be an elliptic curve, where $k$ is an algebraically closed field not of characteristic $p$. Then $\mathscr{K}_{p^{r}}^{\bullet}(E / S) \approx \prod_{G} D_{G}^{\bullet}(k)$ as cochain complexes, where the product runs over all subgroups $G$ of $E\left[p^{\infty}\right] \approx\left(\mathbb{Q}_{p} / \mathbb{Z}_{p}\right)^{\times 2}$ of order $p^{r}$.

Proof This is an explicit combinatorial identification, using the isomorphism of rings

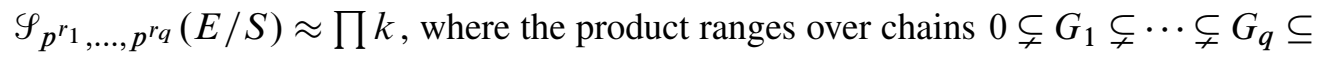
$E\left[p^{\infty}\right] \approx\left(\mathbb{Q}_{p} / \mathbb{Z}_{p}\right)^{2}$ with $\left|G_{q}\right|=p^{r}$.

\section{Supersingular curves, deformations and isogenies}

As noted in Section 1.8, the proof of the main theorem reduces mainly to the special case of a supersingular curve $E_{0} / k$ over a finite field. We will derive this case from the case of the universal deformation $E_{\text {univ }} / k \llbracket x \rrbracket$ of $E_{0} / k$ to rings of characteristic $p$, which we handle by giving a description of the complexes $\mathscr{\kappa}_{p^{r}}^{\bullet}\left(E_{\text {univ }} / k \llbracket x \rrbracket\right)$ using explicit formulas. Although one could consider deformations to rings where $p$ is only assumed to be topological nilpotent, it is not necessary to do so to prove the result we need; in any case I don't know how to construct explicit integral formulas.

In what follows, $E_{0} / k$ will be a fixed supersingular elliptic curve over a perfect field $k$ of characteristic $p$.

For any ring $R$ of characteristic $p$, we write $\sigma=\sigma_{R}: R \rightarrow R$ for the $p$-th power ring endomorphism $\sigma(r)=r^{p}$. For an elliptic curve $E / \operatorname{Spec} R$, we write $E^{\left(p^{r}\right)}=\left(\sigma^{r}\right)^{*} E$. For an element $f(x)=\sum c_{i} x^{i}$ in a power series ring $R \llbracket x \rrbracket$, we will write

$$
f^{\left(p^{r}\right)}=\sum c_{i}^{p^{r}} x^{i} \in R \llbracket x \rrbracket .
$$

\subsection{The category of deformations}

Let $R$ be a local ring of characteristic $p$, and write $k_{R}=R / \mathfrak{m}$. A deformation of $E_{0}$ to $R$ is data $(E, \psi, \alpha)$, where $E$ is an elliptic curve over $\operatorname{Spec} R, \psi: k \rightarrow k_{R}$ is a map of fields, and $\alpha: E \otimes k_{R} \rightarrow \psi^{*} E_{0}$ is an isomorphism of elliptic curves over $\operatorname{Spec} k_{R}$. Let $\left(E_{1}, \psi_{1}, \alpha_{1}\right)$ and $\left(E_{2}, \psi_{2}, \alpha_{2}\right)$ be two deformations of $E_{0}$ to $R$. A deformation of $F^{r}$ is an isogeny $f: E_{1} \rightarrow E_{2}$ of elliptic curves over Spec $R$, such that $\psi_{2}=\psi_{1} \circ \sigma^{r}$, and the square

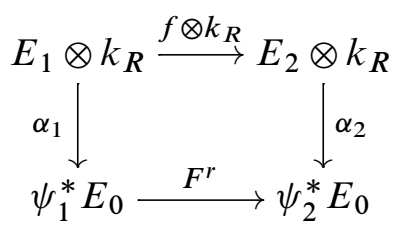


commutes, where $F^{r}$ denotes the $p^{r}$-power relative Frobenius isogeny $F^{r}: \psi_{1}^{*} E_{0} \rightarrow$ $\psi_{1}^{*} E_{0}^{\left(p^{r}\right)}=\psi_{2}^{*} E_{0}$.

A deformation of $F^{r}$ is necessarily a $p^{r}$-isogeny. If $r=0$, we say that $f$ is an isomorphism between deformations.

The collection of all deformations of $E_{0}$ to $R$, and all deformations of $F^{r}$ for $r \geq 0$ between such, forms a category, denoted $\operatorname{Def}(R)=\operatorname{Def}_{E_{0} / k}(R)$.

Example 3.2 Let $(E, \psi, \alpha)$ be a deformation of $E_{0}$ to $R$. Then the Frobenius isogeny $F^{a}: E \rightarrow E^{\left(p^{a}\right)}$ is tautologically a deformation of $F^{a}$; it gives a morphism $(E, \psi, \alpha) \rightarrow\left(E^{\left(p^{a}\right)}, \psi \circ \sigma^{a}, \alpha^{\left(p^{a}\right)}\right)$ in $\operatorname{Def}(R)$.

Any isogeny between deformations of $E_{0}$ factors uniquely through some deformation of $F^{r}$, and so any finite subgroup scheme of rank $p^{r}$ of a deformation of $E_{0}$ is the kernel of an essentially unique deformation of $F^{r}$.

Proposition 3.3 Let $(E, \psi, \alpha)$ be a deformation of $E_{0} / k$ to $R$, and let $G \subset E$ be a subgroup scheme finite and locally free over Spec $R$ of rank $p^{r}$. Then there exists an isogeny $f:(E, \psi, \alpha) \rightarrow\left(E^{\prime}, \psi^{\prime}, \alpha^{\prime}\right)$ which is a deformation of $F^{r}$ and is such that $\operatorname{Ker} f=G$. Given two such isogenies $f_{i}:(E, \psi, \alpha) \rightarrow\left(E_{i}^{\prime}, \psi_{i}^{\prime}, \alpha_{i}^{\prime}\right)$ for $i=1,2$, there exists a unique isomorphism of deformations $g:\left(E_{1}, \psi_{1}, \alpha_{1}\right) \rightarrow\left(E_{2}, \psi_{2}, \alpha_{2}\right)$ such that $g f_{1}=f_{2}$.

Proof Given $G \subset E$, let $E^{\prime}=E / G$ be the quotient curve, defined over $\operatorname{Spec} R$. Passing to $k$, we see that $G_{0}=G \otimes k$ is the unique subgroup scheme of rank $p^{r}$, and thus is the kernel of $F^{r}$. Thus there is a unique isomorphism $\alpha^{\prime}$ making the diagram

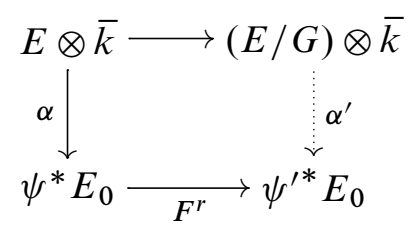

commute, where $\psi^{\prime}=\psi \circ \sigma^{r}$.

The second statement of the proposition is straightforward.

There is at most one deformation of $F^{r}$ (for given $r$ ) between any two deformations.

Proposition 3.4 Let $R$ be an artinian local ring of characteristic $p$. If

$$
f, f^{\prime}:\left(E_{1}, \alpha_{1}, \psi_{1}\right) \rightarrow\left(E_{2}, \alpha_{2}, \psi_{2}\right)
$$

are deformations of $F^{r}$ in $\operatorname{Def}_{E_{0} / k}(R)$, then $f=f^{\prime}$. 
Proof Because $f$ and $f^{\prime}$ are deformations of $F^{r}$, we have that

$$
f \otimes k_{R}=f^{\prime} \otimes k_{R}: E_{1} \otimes k_{R} \rightarrow E_{2} \otimes k_{r}
$$

Thus $\left(f-f^{\prime}\right) \otimes k_{R}$ is the 0 -homomorphism, whence $f-f^{\prime}$ is the 0 -homomorphism by "rigidity" $[4,2.4 .1]$.

\subsection{Universal deformation}

Proposition 3.6 There is at most one isomorphism between any two deformations of $E_{0}$ to $R$. There is a universal deformation $E_{\text {univ }}$ defined over $A \approx k \llbracket x \rrbracket$, with the property that isomorphism classes of deformations of $E_{0}$ to an artinian local ring $R$ of characteristic $p$ are in bijective correspondence with local homomorphisms of rings $A \rightarrow R$.

Proof This is a standard result of deformation theory. The Serre-Tate theorem says that deformations of $E_{0}$ are the same as deformations of its underlying formal group $\widehat{E}_{0}$, which is a formal group of height 2 , and the deformations of such formal groups are classified by a theorem of Lubin and Tate.

Thus, the isomorphism class of a deformation $(E, \alpha, \psi)$ of $E_{0}$ to $R$ corresponds, to a unique local ring homomorphism $\phi_{(E, \alpha, \psi)}: A \rightarrow R$. If we make a choice of generator $x \in A$, so that $A \approx k \llbracket x \rrbracket$, then we can speak of the deformation parameter $x(E, \alpha, \psi):=\phi_{(E, \alpha, \psi)}(x) \in \mathfrak{m}_{R}$. Thus, deformations of $(E, \alpha, \psi)$ to an artinian local ring $R$ correspond up to isomorphism to pairs $(\psi, a)$ consisting of a ring homomorphism $\psi: k \rightarrow k_{R}$ and an element $a \in \mathfrak{m}_{R}$, where $a=x(E, \alpha, \psi)$.

If two deformations are related by a Frobenius isogeny, their deformation parameters are related in an obvious way.

Proposition 3.7 Let $R$ be an artinian local ring of characteristic $p$. Denote the $p^{a}$ power Frobenius by $F^{a}:(E, \alpha, \psi) \rightarrow\left(E^{\left(p^{a}\right)}, \alpha^{\left(p^{a}\right)}, \psi \circ \sigma^{a}\right)$ (viewed as a morphism between deformations of $E_{0} / k$ to $R$ ). Then we have that

$$
\phi_{\left(E^{\left(p^{a}\right)}, \alpha^{\left(p^{a}\right)}, \psi \circ \sigma^{a}\right)}=\phi_{(E, \alpha, \psi)} \circ \sigma^{a}: A \rightarrow R .
$$

Proof Immediate. 


\subsection{Standard supersingular curves over $\mathbb{F}_{p^{2}}$}

We'll say that a supersingular elliptic curve $E_{0} / k$ is standard if $k=\mathbb{F}_{p^{2}}$ and $F^{2}=[-p]$. Thus, to prove case (C) of Section 1.8, it will suffice to prove it in the case of standard supersingular curves, by means of the following.

Proposition 3.9 Every supersingular curve over a field containing $\mathbb{F}_{p^{2}}$ is isomorphic to some standard curve $E_{0} / \mathbb{F}_{p^{2}}$.

Proof That all supersingular curves have models over $\mathbb{F}_{p^{2}}$ is well known. The statement about the $p^{2}$-power Frobenius is proved by Baker et al [1, Lemma 3.21]. See also the discussion [5].

For an elliptic curve $E$ over a ring $R$ of characteristic $p$, we write $V^{b}=V_{E}^{b}: E^{\left(p^{b}\right)} \rightarrow E$ for the $p^{b}$-power Verschiebung isogeny, defined as the dual of the $p^{b}$-power Frobenius $F_{E}^{b}: E \rightarrow E^{\left(p^{b}\right)}$. We write $(-V)^{b}=\left(-V_{E}\right)^{b}: E^{\left(p^{b}\right)} \rightarrow E$ for the composite $V_{E}^{b} \circ\left[(-1)^{b}\right]_{E^{\left(p^{b}\right)}}: E^{\left(p^{b}\right)} \rightarrow E$. Our interest in standard supersingular curves comes from the following.

Proposition 3.10 Given a deformation $(E, \alpha, \psi)$ of a standard supersingular curve $E_{0} / \mathbb{F}_{p^{2}}$ to $R$, the isogeny $\left(-V_{E}\right)^{b}$ is a deformation of $F^{b}$. That is,

$$
\left(-V_{E}\right)^{b}:\left(E^{\left(p^{b}\right)}, \alpha^{\left(p^{b}\right)}, \psi \circ \sigma^{b}\right) \rightarrow(E, \alpha, \psi)
$$

is a morphism in $\operatorname{Def}(R)$. In this case we have that

$$
\phi_{\left(E^{\left.\left(p^{b}\right), \alpha^{\left(p^{b}\right)}, \psi \circ \sigma^{b}\right)}\right.}=\phi_{(E, \alpha, \psi)} \circ \sigma^{b}: A \rightarrow R .
$$

Proof Since $E_{0}$ is defined over $\mathbb{F}_{p^{2}}$, we have $E_{0}^{\left(p^{2 r}\right)}=E_{0}$ for all $r$. Since $E_{0} / \mathbb{F}_{p^{2}}$ is a standard supersingular curve, we have that $F^{2}=-p=-V F$ on $E_{0}$, and therefore that

$$
\left(-V_{E_{0}}\right)^{b}=F_{E_{0}^{(p)}}^{b}: E_{0}^{\left(p^{b}\right)} \rightarrow E_{0}^{\left(p^{2 b}\right)}=E_{0} .
$$

Thus, the commutative diagram of elliptic curves and isogenies over $k_{R}$

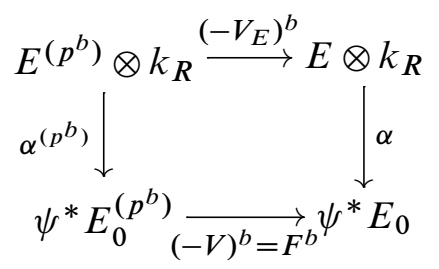

shows that $\left(-V_{E}\right)^{b}$ is a deformation of $F^{b}$. 


\subsection{Isogenies of type $(a, b)$}

Let $S$ be an $\mathbb{F}_{p}$-scheme, and let $E_{1}$ and $E_{2}$ be elliptic curves over $S$. An isogeny of type $(a, b)[4,13.3 .4]$ is a $p^{a+b}$-isogeny $f: E_{1} \rightarrow E_{2}$ of curves over $S$ which admits a factorization of the form

$$
E_{1} \stackrel{F^{a}}{\longrightarrow} E_{1}^{\left(p^{a}\right)} \stackrel{g}{\sim} E_{2}^{\left(p^{b}\right)} \stackrel{V^{b}}{\longrightarrow} E_{2},
$$

where $g$ is an isomorphism. Equivalently, $f$ is of type $(a, b)$ if it admits a factorization of the form

$$
E_{1} \stackrel{F^{a}}{\longrightarrow} E_{1}^{\left(p^{a}\right)} \stackrel{g^{\prime}}{\longrightarrow} E_{2}^{\left(p^{b}\right)} \stackrel{(-V)^{b}}{\longrightarrow} E_{2} .
$$

Proposition 3.12 Let $E_{0} / k$ be a standard supersingular elliptic curve, and let $\left(E_{1}, \alpha_{1}, \psi_{1}\right)$ and $\left(E_{2}, \alpha_{2}, \psi_{2}\right)$ be two deformations of $E_{0}$ to an artinian local $\mathbb{F}_{p}-$ algebra $R$. Suppose $r=a+b$. The following are equivalent.

(1) There exists a (necessarily unique) isogeny $f: E_{1} \rightarrow E_{2}$ which is (i) a deformation of $F^{r}$, and (ii) of type $(a, b)$.

(2) $\phi_{\left(E_{1}, \alpha_{1}, \psi_{1}\right)} \circ \sigma^{a}=\phi_{\left(E_{2}, \alpha_{2}, \psi_{2}\right)} \circ \sigma^{b}$ as maps $A \rightarrow R$.

Proof Suppose $\phi_{\left(E_{1}, \alpha_{1}, \psi_{1}\right)} \circ \sigma^{a}=\phi_{\left(E_{2}, \alpha_{2}, \psi_{2}\right)} \circ \sigma^{b}$, which means that there exists an isomorphism $g:\left(E^{\left(p^{a}\right)}, \alpha_{1}^{\left(p^{a}\right)}, \psi_{1}\right) \rightarrow\left(E^{\left(p^{b}\right)}, \alpha_{2}^{\left(p^{b}\right)}, \psi_{2}\right)$ in $\operatorname{Def}(R)$. Then $(-V)^{b} \circ g \circ F^{a}: E_{1} \rightarrow E_{2}$ is a deformation of $F^{r}$ (using Proposition 3.10) and an isogeny of type $(a, b)$.

Conversely, consider a deformation of $F^{r}$ of the form $f=(-V)^{b} \circ g \circ F^{a}: E_{1} \rightarrow E_{2}$. In the diagram

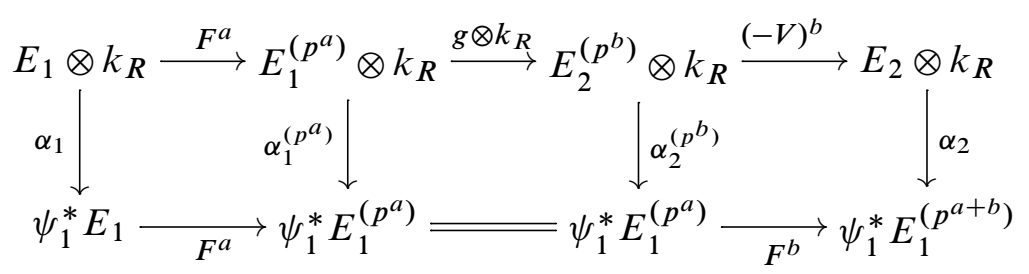

the left-hand and right-hand squares, as well as the large rectangle, commute. Therefore we must have

$$
\alpha_{1}^{\left(p^{a}\right)}=\alpha_{2}^{\left(p^{b}\right)} \circ\left(g \otimes k_{R}\right),
$$

whence $\left(E_{1}^{\left(p^{a}\right)}, \alpha_{1}^{\left(p^{a}\right)}, \psi_{1} \circ \sigma^{a}\right)$ and $\left(E_{2}^{\left(p^{b}\right)}, \alpha_{2}^{\left(p^{b}\right)}, \psi_{2} \circ \sigma^{b}\right)$ are isomorphic deformations.

Given a choice of generator $x \in A$, we can restate this as follows. 
Corollary 3.13 Let $E_{0} / k$ be a standard supersingular curve. Let $\left(E_{1}, \alpha_{1}, \psi_{1}\right)$ and $\left(E_{2}, \alpha_{2}, \psi_{2}\right)$ be two objects of $\operatorname{Def}_{E_{0} / k}(R)$, with deformation parameters $x_{i}=x\left(E_{i}, \alpha_{i}, \psi_{i}\right) \in R$ for $i=1,2$. There exists a (necessarily unique) morphism $f:\left(E_{1}, \alpha_{1}, \psi_{1}\right) \rightarrow\left(E_{2}, \alpha_{2}, \psi_{2}\right)$ in $\operatorname{Def}_{E_{0} / k}(R)$ of type $(a, b)$ if and only if

(i) $\psi_{2}=\psi_{1} \circ \sigma^{a+b}$, and

(ii) $x_{1}^{p^{a}}=x_{2}^{p^{b}}$.

Proof The only thing to note is that since $k=\mathbb{F}_{p^{2}}$, (i) is equivalent to $\psi_{1} \circ \sigma^{a}=$ $\psi_{2} \circ \sigma^{b}$.

\subsection{Explicit description of the deformation category of a standard super- singular curve}

As before, $E_{0} / k$ is a standard supersingular curve.

Let $F_{p^{r}}(x, y) \in k[x, y]$ be the polynomial given by

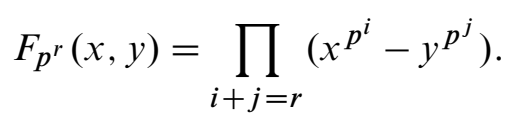

Proposition 3.15 Let $\left(E_{1}, \alpha_{1}, \psi_{1}\right)$ and $\left(E_{2}, \alpha_{2}, \psi_{2}\right)$ be two deformations of $E_{0}$ to an artinian local $\mathbb{F}_{p}$-algebra $R$, with deformation parameters $x_{i}=x\left(E_{i}, \alpha_{i}, \psi_{i}\right) \in R$. There exists a (necessarily unique) deformation of $F^{r}$ from $\left(E_{1}, \alpha_{1}, \psi_{1}\right)$ to $\left(E_{2}, \alpha_{2}, \psi_{2}\right)$ if and only if

(i) $\psi_{2}=\psi_{1} \circ \sigma^{r}$, and

(ii) $F_{p^{r}}\left(x_{1}, x_{2}\right)=0$.

Moreover, there is a universal example of a deformation of $F^{r}$ given by $f: s^{*} E_{\text {univ }} \rightarrow$ $t^{*} E_{\text {univ }}$, defined over the ring $A_{r} \approx k \llbracket x_{1}, x_{2} \rrbracket /\left(F_{p^{r}}\left(x_{1}, x_{2}\right)\right)$ with $s, t: A \approx k \llbracket x \rrbracket \rightarrow A_{r}$ given by $s(f(x))=f\left(x_{1}\right)$ and $t(f(x))=f^{\left(p^{r}\right)}\left(x_{2}\right)$.

Remark 3.16 Consider a different choice $x^{\prime} \in k \llbracket x \rrbracket$ of deformation parameter, so that $x^{\prime}=f(x)=c_{1} x+\cdots \in k \llbracket x \rrbracket$ with $c_{1} \neq 0$, and let $x_{1}^{\prime}=f\left(x_{1}\right)$ and $x_{2}^{\prime}=f^{\left(p^{r}\right)}\left(x_{2}\right)$ in $k \llbracket x_{1}, x_{2} \rrbracket$. Because $k=\mathbb{F}_{p^{2}}$, we have that $f^{\left(p^{2 r-l}\right)}(x)=f^{\left(p^{i}\right)}(x)$, and thus

$$
\begin{aligned}
x_{1}^{\prime p^{i}}-x_{2}^{\prime p^{r-i}}=f\left(x_{1}\right)^{p^{i}}-f^{\left(p^{r}\right)}\left(x_{2}\right)^{p^{r-i}} & =f^{\left(p^{i}\right)}\left(x_{1}^{p^{i}}\right)-f^{\left(p^{i}\right)}\left(x_{2}^{p^{r-i}}\right) \\
& \left.=\left(x_{1}^{p^{i}}-x_{2}^{p^{r-i}}\right) \text { (unit in } k \llbracket x_{1}, x_{2} \rrbracket\right) .
\end{aligned}
$$

Thus, the elements $F_{p^{r}}\left(x_{1}, x_{2}\right)$ and $F_{p^{r}}\left(x_{1}^{\prime}, x_{2}^{\prime}\right)$ generate the same ideal in $k \llbracket x_{1}, x_{2} \rrbracket$; our description of $A_{r}$ does not depend on the choice of deformation parameter. 
Proof We have already noted that giving a deformation of $F^{r}$ with given domain $\left(E_{1}, \alpha_{1}, \psi_{1}\right)$ is the same as giving a subgroup scheme of order $p^{r}$. According to the discussion in [4, Section 6.8, (especially pages 181-183)], the universal example of such a subgroup scheme $G$ of a deformation $E$ of $E_{0}$ is defined over a ring of the form $A_{r}=k \llbracket x_{1}, x_{2} \rrbracket / J$, where $J$ is a principal ideal; $x_{1}$ and $x_{2}$ are the deformation parameters of $E$ and $E / G$ respectively. Thus, it suffices to describe a generator $g$ of $J$. That we can take $g=F_{p^{r}}\left(x_{1}, x_{2}\right)$ is the essential content of $[4,13.4 .6]$, which is an application of the "crossings theorem" $[4,13.1 .3]$.

We can give a quick and dirty proof that $J=\left(F_{p^{r}}\left(x_{1}, x_{2}\right)\right)$. As noted, we can write $J=(g)$ for some element $g$. For $a+b=r$ the projection map

$$
\gamma_{a b}: A_{r}=k \llbracket x_{1}, x_{2} \rrbracket /(g) \rightarrow k \llbracket x_{1}, x_{2} \rrbracket /\left(x_{1}^{p^{a}}-x_{2}^{p^{b}}\right),
$$

is precisely the ring homomorphism which classifies the universal deformation of $F^{r}$ of type $(a, b)$. For each $a+b=r$ write $g_{a b}=x_{1}^{p^{a}}-x_{2}^{p^{b}}$; the existence of $\gamma_{a b}$ shows that $g_{a b}$ divides $g$. We have that $g_{a b}=f_{a b}^{p^{\min (a, b)}}$, where $f_{a b}$ is an irreducible element of $k \llbracket x, y \rrbracket$, and any pair of the $f_{a b}$ 's are distinct-up-to-units. Thus, since $k \llbracket x_{1}, x_{2} \rrbracket$ is a UFD, the product $F_{p^{r}}\left(x_{1}, x_{2}\right)=\prod g_{a, b}$ must also divide $g$. We know that $A_{r}$ (since it classifies subgroup schemes of order $p^{r}$ ) is finite and free over $k \llbracket x_{1} \rrbracket$ of rank $1+p+\cdots+p^{r} ;$ thus

$$
g \equiv x_{2}^{1+p+\cdots+p^{r}} \cdot(\text { unit }) \equiv F_{p^{r}}\left(x_{1}, x_{2}\right) \cdot(\text { unit }) \bmod \left(x_{1}\right),
$$

and so $g=F_{p^{r}}\left(x_{1}, x_{2}\right) \cdot$ (unit) by Weierstrass preparation.

As a result of the above proposition, the category $\operatorname{Def}(R)$ of deformations of a standard supersingular curve to an artinian local $\mathbb{F}_{p}$-algebra is equivalent to the category in which

(1) objects are pairs $(\psi, a)$ consisting of ring homomorphisms $\psi: k \rightarrow k_{R}$ and elements $a \in \mathfrak{m}_{R}$, and

(2) morphisms $\left(\psi_{1}, a_{1}\right) \rightarrow\left(\psi_{2}, a_{2}\right)$ are integers $r \geq 0$ such that $\psi_{2}=\psi_{1} \circ \sigma^{r}$ and $F_{p^{r}}\left(a_{1}, a_{2}\right)=\prod_{i+j=r}\left(a_{1}^{p^{i}}-a_{2}^{p^{j}}\right)=0$ in $R$.

It is not a priori obvious that composition in the above category well-defined (though it must be by Proposition 3.15), since this would amount to showing that $F_{p^{r}}(a, b)=0$ and $F_{p^{r^{\prime}}}(b, c)=0$ imply $F_{p^{r+r^{\prime}}}(a, c)=0$ for $a, b, c \in \mathfrak{m}_{R}$. In the Appendix we give a direct proof of this fact about these polynomials. 


\subsection{Explicit description of $\mathscr{K}_{p^{r}}^{\bullet}$ for universal deformations of a supersin- gular curve}

Fix a universal deformation $E / S$ of a standard supersingular curve $E_{0} / k$, where $S=\operatorname{Spec} A$. Fix an isomorphism $A=k \llbracket x \rrbracket$, and write $A_{r}=\mathscr{Y}_{p^{r}}(E / S)$, and more generally $A_{r_{1}, \ldots, r_{q}}=\mathscr{S}_{p^{r_{1}, \ldots, p^{r q}}}(E / S)$.

The discussion of Section 3.14 can be summarized as follows.

Proposition 3.18 Let $s, t: A \rightarrow A_{r}$ be the maps classifying respectively the source and target of the universal deformation of $F^{r}$, as in Proposition 3.15.

(1) There are isomorphisms

$$
A_{r} \approx k \llbracket x_{0}, x_{1} \rrbracket /\left(F_{p^{r}}\left(x_{0}, x_{1}\right)\right),
$$

such that $s: A \rightarrow A_{r}$ is given by $s(f(x))=f\left(x_{0}\right)$ and $t: A \rightarrow A_{r}$ is given by $t(f(x))=f^{\left(p^{r}\right)}\left(x_{1}\right)$.

(2) There are isomorphisms

$$
\begin{aligned}
A_{r_{1}, \ldots, r_{q}} & \approx A_{r_{1}}{ }^{t} \otimes_{A}{ }^{s} \ldots{ }^{t} \otimes_{A}{ }^{s} A_{r_{q}} \\
& \approx k \llbracket x_{0}, \ldots, x_{q} \rrbracket\left(F_{p^{r_{1}}}\left(x_{0}, x_{1}\right), \ldots, F_{p^{r_{q}}}\left(x_{q-1}, x_{q}\right)\right),
\end{aligned}
$$

where the map $s_{k}: A \rightarrow A_{r_{1}, \ldots, r_{q}}$ given by $s_{k}(f(x))=f^{\left(p^{r_{1}+\cdots+r_{k}}\right)}\left(x_{k}\right)$ classifies the quotient curve $E / G_{k}$ (in the notation of Section 1.3).

(3) With respect to the above isomorphism, the map $u_{k}: A_{r_{1}, \ldots, r_{k-1}+r_{k}, \ldots, r_{q}} \rightarrow$ $A_{r_{1}, \ldots, r_{q}}$ is given by $u_{k}\left(x_{i}\right)=x_{i}$ if $i<k$, and $u_{k}\left(x_{i}\right)=x_{i+1}$ if $i \geq k$.

This determines explicitly the structure of the complex $\mathscr{K}_{p^{r}}^{\bullet}(E / S)$. Thus, to prove case (C) of Section 1.8, it suffices to prove (1) and (2) of Theorem 1.6 for this explicit complex.

\subsection{Two useful lemmas}

The following two lemmas will be needed in the next section.

Lemma 3.20 For all $r \geq 1$, the homomorphism $u_{1}: A_{r+1} \rightarrow A_{1, r}$ is the inclusion of an $A$-module summand, where we regard $A_{r+1}$ as an $A$-module by $s: A \rightarrow A_{r+1}$. 
Proof By Nakayama's lemma it is enough to prove that the map $u_{1}$ is injective after tensoring down along $A \approx k \llbracket x \rrbracket \rightarrow k$. Thus, it suffices to show that the ring homomorphism

$$
k \llbracket z \rrbracket /\left(z^{1+p+\cdots+p^{r+1}}\right) \rightarrow k \llbracket y, z \rrbracket /\left(y^{1+p}, F_{p^{r}}(y, z)\right)=B
$$

sending $z \mapsto z$ is injective. It will suffice to show that $z^{p+\cdots+p^{r+1}} \neq 0$ in $B$. Observe that $B$ has a basis over $k$ given by the monomials $y^{i} z^{j}$ with $0 \leq i \leq p$ and $0 \leq j \leq$ $p+p^{2}+\cdots+p^{r}$.

In the target ring $B$ we have

$$
\begin{aligned}
0=F_{p^{r}}(y, z)^{p} & =\left(y-z^{p^{r}}\right)^{p}\left(y^{p}-z^{p^{r-1}}\right)^{p}\left(y^{p^{2}}-z^{p^{r-2}}\right)^{p} \cdots\left(y^{p^{r}}-z\right)^{p} \\
& = \pm\left(y^{p}-z^{p^{r+1}}\right) z^{p^{r}} z^{p^{r-1}} \cdots z^{p}
\end{aligned}
$$

and thus $z^{p+p^{2}+\cdots+p^{r+1}}=y^{p} z^{p+p^{2}+\cdots+p^{r}}$ is one of the elements of our $k$-basis for $B$, and thus is nonzero.

Lemma 3.21 There is a split short exact sequence of $A$-modules

$$
0 \rightarrow A_{2} \stackrel{u_{1}}{\longrightarrow} A_{1,1} \stackrel{\bar{v}}{\rightarrow} A_{1} / s(A) \rightarrow 0
$$

where $\bar{v}$ is the composition of the projection $A_{1} \rightarrow A_{1} / s(A)$ with the ring homomorphism $v: A_{1,1} \rightarrow A_{1}$ defined by

$$
v\left(x_{0}\right)=x_{0}=v\left(x_{2}\right), \quad v\left(x_{1}\right)=x_{1},
$$

using the identifications $A_{1,1}=k \llbracket x_{0}, x_{1}, x_{2} \rrbracket /\left(F_{p}\left(x_{0}, x_{1}\right), F_{p}\left(x_{1}, x_{2}\right)\right)$ and $A_{1}=$ $k \llbracket x_{0}, x_{1} \rrbracket /\left(F_{p}\left(x_{0}, x_{1}\right)\right)$ of Proposition 3.15.

Proof Consider the commutative square of ring maps

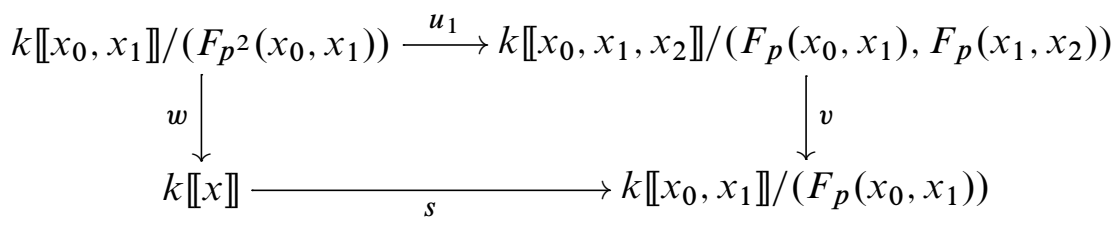

where $w\left(x_{0}\right)=x=w\left(x_{1}\right)$. The horizontal maps are injective, and the vertical maps are surjective, and the induced map of cokernels Cok $u_{1} \rightarrow \operatorname{Cok} s$ is a surjective map between free $A=k \llbracket x_{0} \rrbracket$-modules of rank $p$, and thus is an isomorphism. 


\section{The proof of the theorem for supersingular curves}

Recall that we wish to prove statements (1) and (2) of Theorem 1.6 for a standard supersingular curve $E_{0} / k$. It is clear that it suffices to prove these statements for the universal deformation $E / S$, where $S=\operatorname{Spec} A$ with $A=k \llbracket x \rrbracket$. Thus, from now on we fix such a universal deformation $E / S$.

By Section 3.17, we have obtained an explicit description of the complexes $\mathscr{K}_{p^{r}}^{\bullet}(E / S)$. Thus, we will prove the desired results by means of an explicit calculation.

\subsection{A dual formulation}

We will not work directly with the complex $\mathscr{K}_{p^{r}}^{\bullet}(E / S)$, but rather with its dual. Let $K_{\bullet}^{r}$ be the chain complex which is $A$-linear dual to $\mathscr{K}_{p^{r}}^{\bullet}(E / S)$. Thus $K_{q}^{r}=$ $\operatorname{Hom}_{A}\left(\mathscr{K}_{p^{r}}^{q^{r}}(E / S), A\right)$, where the $A$-module structure on $\mathscr{K}_{p^{r}}^{q^{r}}(E / S)$ (and thus on $K_{q}^{r}$ ) is induced by the ring homomorphisms $s: A \rightarrow A_{r_{1}, \ldots, r_{q}}$. To prove $H^{j}\left(\mathcal{K}_{p^{r}}^{\bullet}(E / S)\right)=0$ for $j \neq r$ and is a projective $A$-module for $j=r$, we will use the following observation.

Proposition 4.2 Let $A$ be a commutative ring and let $P^{\bullet}=\left(0 \rightarrow P^{0} \rightarrow \cdots \rightarrow P^{n} \rightarrow 0\right)$ be a bounded cochain complex of finitely generated projective $A$-modules. Let $P_{\bullet}=$ $\operatorname{Hom}_{A}\left(P^{\bullet}, A\right)$ denote the chain complex obtained by taking $A$-linear duals. Then the following are equivalent.

(1) $H^{j}\left(P^{\bullet}\right)=0$ for $j \neq n$, and $H^{n}\left(P^{\bullet}\right)$ is a finitely generated projective $A-$ module.

(2) $H_{j}\left(P_{\bullet}\right)=0$ for $j \neq n$.

Proof Condition (1) says that the sequence $0 \rightarrow P^{0} \rightarrow \cdots \rightarrow P^{n} \rightarrow H^{n}\left(P^{\bullet}\right) \rightarrow 0$ is an exact sequence of finitely generated projective modules. Condition (2) says that the sequence $0 \rightarrow H_{n}\left(P_{\bullet}\right) \rightarrow P_{n} \rightarrow \cdots \rightarrow P_{0} \rightarrow 0$ is a exact sequence of modules, all of which are finitely generated projective except perhaps for $H_{n}\left(P_{\bullet}\right)$; but then it is straightforward to show that $H_{n}\left(P_{\bullet}\right)$ must be projective and finitely generated as well. The result then follows from the fact that $\operatorname{Hom}_{A}(-, A)$ is a contravariant autoequivalence of the category of finitely generated projectives.

Thus, it will suffice to prove that $H_{j} K_{\bullet}^{r}=0$ for $j \neq r$. The remainder of the section is devoted to the proof of this Proposition 4.20. 


\subsection{Bimodules and duals}

We establish some notation. Fix a commutative ring $A$. Given an $A$-bimodule $M$, we write $M^{*}$ for the set $\operatorname{Hom}_{A}(M, A)$ of left $A$-module homomorphisms. Then $M^{*}$ admits the structure of an $A$-bimodule, defined by

$$
(a \cdot \phi \cdot b)(m)=\phi(a \cdot m \cdot b)
$$

for $a, b \in A, m \in M, \phi \in M^{*}$.

Given $A$-bimodules $M$ and $N$, we define a map

$$
M^{*} \otimes_{A} N^{*} \rightarrow\left(M \otimes_{A} N\right)^{*}
$$

of $A$-bimodules by

$$
(\phi \otimes \psi)(m \otimes n)=\phi(m \cdot \psi(n)) .
$$

(One must check that this is well-defined, and actually gives a map of bimodules. Note that $A$ is commutative; the formulas we use here don't make sense for noncommutative $A$.)

More generally, we obtain $A$-bimodule maps

$$
M_{1}^{*} \otimes_{A} \cdots \otimes_{A} M_{q}^{*} \rightarrow\left(M_{1} \otimes_{A} \cdots \otimes_{A} M_{q}\right)^{*}
$$

by

$$
\left(\phi_{1} \otimes \cdots \otimes \phi_{q}\right)\left(m_{1} \otimes \cdots m_{q}\right)=\phi_{1}\left(m_{1} \cdot \phi_{2}\left(m_{2} \cdots \phi_{q}\left(m_{q}\right)\right)\right) .
$$

We note that if each $M_{i}$ is finitely generated and free as a left $A$-module, then so is $M_{1} \otimes_{A} \cdots \otimes_{A} M_{q}$, and the above map is an isomorphism.

\subsection{The ring $\Gamma$}

We will describe the dual complex $K_{\bullet}^{r}$ in terms of a certain graded associative ring $\Gamma=\bigoplus_{r \geq 0} \Gamma_{r}$. This ring will contain $A=k \llbracket x \rrbracket$ (in fact, $\Gamma_{0}=A$ ), but $A$ will not be central in $\Gamma$.

We will regard each ring $A_{r}=\mathscr{S}_{p^{r}}(E / S)$ as an $A$-bimodule, with the left $A$-module structure coming from $s: A \rightarrow A_{r}$, and the right $A$-module structure coming from $t: A \rightarrow A_{r}$. With this notation we have an isomorphism of $A$-bimodules $A_{r_{1}, \ldots, r_{q}}=$ $A_{r_{1}} \otimes_{A} \cdots \otimes_{A} A_{r_{q}}$, and each of the maps $u_{i}: A_{r_{1}, \ldots, r_{i-1}+r_{i}, \ldots, r_{q}} \rightarrow A_{r_{1}, \ldots, r_{q}}$ is thus an $A$-bimodule homomorphism.

Let $\Gamma_{r}=A_{r}^{*}$. As we have observed, $\Gamma_{r}$ is naturally an $A$-bimodule. In terms of explicit power series, the bimodule structure is defined by

$$
(f(x) \cdot \phi \cdot g(x))\left(h\left(x_{0}, x_{1}\right)\right)=\phi\left(f\left(x_{0}\right) h\left(x_{0}, x_{1}\right) g^{\left(p^{r}\right)}\left(x_{1}\right)\right) .
$$


Observe that $\Gamma_{0}=A^{*} \approx A$, and that since $A_{r}$ is finitely generated and free as a left $A$-module, so is $\Gamma_{r}$.

From the above remarks, we see that the $A$-bimodule isomorphism $A_{r} \otimes_{A} A_{r^{\prime}} \approx A_{r, r^{\prime}}$ gives an isomorphism $\Gamma_{r} \otimes_{A} \Gamma_{r^{\prime}} \rightarrow A_{r, r^{\prime}}^{*}$.

Define a product $\mu: \Gamma_{r} \otimes_{A} \Gamma_{r^{\prime}} \rightarrow \Gamma_{r+r^{\prime}}$ by

$$
(\mu(\phi \otimes \psi))(g)=(\phi \otimes \psi)\left(u_{1}(g)\right),
$$

where $\phi \in \Gamma_{r}, \psi \in \Gamma_{r^{\prime}}$, and $g \in A_{r+r^{\prime}}$. That is, $\mu$ is dual to the $A$-bimodule map $u_{1}: A_{r+r^{\prime}} \rightarrow A_{r, r^{\prime}}$. This makes $\Gamma=\bigoplus_{r} \Gamma_{r}$ into a graded associative ring, which contains the ring $A=\Gamma_{0}$.

Proposition 4.5 For all $r \geq 1$, the product map $\mu: \Gamma_{1} \otimes_{A} \Gamma_{r-1} \rightarrow \Gamma_{r}$ is surjective. In particular, $\Gamma$ is generated as a ring by $\Gamma_{0}$ and $\Gamma_{1}$.

Proof Immediate using Lemma 3.20.

\subsection{The complex $K_{\bullet}^{r}$ is a bar resolution of $\Gamma$}

We thus have the following description of the complex $K_{\bullet}^{r}$.

Proposition 4.7 For $r \geq 1$, there are isomorphisms of $A$-modules

$$
K_{q}^{r} \approx \bigoplus_{r_{1}+\cdots+r_{q}=r} \Gamma_{r_{1}} \otimes_{A} \cdots \otimes_{A} \Gamma_{r_{q}}
$$

where the sum is taken over tuples $\left(r_{1}, \ldots, r_{q}\right)$ of positive integers which sum to $r$. With respect to these isomorphisms, the boundary map $\partial: K_{q}^{r} \rightarrow K_{q-1}^{r}$ is given by

$$
\partial\left(\phi_{1} \otimes \cdots \otimes \phi_{r}\right)=\sum_{i=1}^{q-1}(-1)^{i} \phi_{1} \otimes \cdots \otimes \phi_{i} \phi_{i+1} \otimes \cdots \phi_{q},
$$

where $\phi_{1} \otimes \cdots \otimes \phi_{r} \in \Gamma_{r_{1}} \otimes_{A} \cdots \otimes_{A} \Gamma_{r_{q}} \subseteq K_{q}^{r}$, and $\phi_{1} \otimes \cdots \otimes \phi_{i} \phi_{i+1} \otimes_{A} \cdots \otimes_{A} \phi_{q} \in$ $\Gamma_{r_{1}} \otimes_{A} \cdots \otimes_{A} \Gamma_{r_{i}+r_{i+1}} \otimes_{A} \cdots \otimes_{A} \Gamma_{r_{q}} \subseteq K_{q-1}^{r}$.

Proof Immediate.

This amounts to saying that the complex $K_{\bullet}=\bigoplus_{r} K_{\bullet}^{r}$ is isomorphic to the normalized bar complex $\overline{\mathcal{B}}(A, \Gamma, A)$ of the augmented associative ring $\Gamma$. 


\subsection{Relations in $\Gamma$}

We now describe certain elements $P_{i}$ in $\Gamma$, and certain relations among them; below it will be shown that this gives a presentation of $\Gamma$ in terms of generators and relations.

For $0 \leq i \leq p$, let $P_{i} \in \Gamma_{1}$ denote the element defined by

$$
P_{i}\left(x_{1}^{j}\right)=0 \text { if } i \neq j, \quad P_{i}\left(x_{1}^{i}\right)=1,
$$

where $0 \leq j \leq p$. That is, $P_{0}, \ldots, P_{p}$ is a left $A$-module basis of $\Gamma_{1}$, dual to the monomial left $A$-module basis $1, x_{1}, \ldots, x_{1}^{p}$ of $A_{1}=k \llbracket x_{0}, x_{1} \rrbracket /\left(F_{p}\left(x_{0}, x_{1}\right)\right)$.

The right $A$-module structure on $\Gamma_{1}$ may be described as follows. A straightforward calculation shows that for $c \in k$,

$$
P_{i} c=c^{p} P_{i},
$$

and for the generator $x \in k \llbracket x \rrbracket=A$, we have

$$
\begin{aligned}
& P_{0} x=-x^{p+1} P_{p}, \\
& P_{1} x=P_{0}+x P_{p}, \\
& P_{i} x=P_{i-1} \quad(\text { if } 1<i<p), \\
& P_{p} x=P_{p-1}+x^{p} P_{p} .
\end{aligned}
$$

(This amounts to the identity $x_{1}^{p+1}=-x_{0}^{p+1}+x_{0} x_{1}+x_{0}^{p} x_{1}^{p}$ in $A_{1}$.)

Observe that these imply that for each $i=0, \ldots, p$, we have $P_{i} x^{p+1}=x Q_{i}$ for some element $Q_{i} \in \Gamma_{1}$. Thus, the above identities determine the structure of $\Gamma_{1}$ as a right $A$-module; for, if $f(x) \in k \llbracket x \rrbracket$ is a limit of a sequence of polynomials $f_{n}(x)$, we see that $P_{i} f(x)$ is the limit as $n \rightarrow \infty$ of the sequence $\left\{P_{i} f_{n}(x)\right\}$ with respect to the $x$-adic topology.

The exact sequence of Lemma 3.21 gives rise, on taking duals, to a short exact sequence

$$
0 \rightarrow\left(A_{1} / s(A)\right)^{*} \stackrel{\bar{v}^{*}}{\rightarrow} \Gamma_{1} \otimes_{A} \Gamma_{1} \stackrel{\mu}{\rightarrow} \Gamma_{2} \rightarrow 0 .
$$

The natural inclusion $\left(A_{1} / s(A)\right)^{*} \subset A_{1}^{*} \approx \Gamma_{1}$ identifies $\left(A_{1} / s(A)\right)^{*}$ with the left sub- $A$-module of $\Gamma_{1}$ spanned by $P_{1}, \ldots, P_{p}$, and a straightforward calculation shows that $\bar{v}^{*}\left(P_{i}\right)=\sum_{j=0}^{p} x^{j} P_{i} \otimes P_{j} \in \Gamma_{1} \otimes_{A} \Gamma_{1}$. That is, the identity

$$
P_{i} P_{0}+x P_{i} P_{1}+\cdots+x^{p} P_{i} P_{p}=0
$$

holds in the ring $\Gamma$ for each $i=1, \ldots, p$. (It does not hold for $i=0$.) 


\subsection{The structure of $\Gamma$}

Denote the tensor algebra on the $A$-bimodule $\Gamma_{1}$ by

$$
T \Gamma_{1}=\bigoplus_{r \geq 0} \underbrace{\Gamma_{1} \otimes_{A} \cdots \otimes_{A} \Gamma_{1}}_{r \text { factors }} .
$$

Let $\Delta=T \Gamma_{1} / J$, where $J$ is the two-sided ideal generated by $\sum_{j=0}^{p} x^{j} P_{i} P_{j}$ for $i=1, \ldots, p$. Observe that since $J$ is generated by homogeneous elements, we have $\Delta \approx \bigoplus_{r \geq 0} \Delta_{r}$ where $\Delta_{r}$ is an $A$-bimodule quotient of $\Gamma_{1}^{\otimes_{A} r}$.

A sequence will be a list $I=\left(i_{1}, \ldots, i_{r}\right)$, of length $r \geq 0$, of elements of $\{0,1, \ldots, p\}$. We say such a sequence is inadmissible if there exists a $k$ such that $i_{k} \neq 0$ and $i_{k+1}=0$; otherwise, it is admissible. Thus, a sequence is admissible precisely if all zeros in $I$ appear at the beginning of the sequence.

Given a sequence $I=\left(i_{1}, \ldots, i_{r}\right)$, we write $P_{I}=P_{i_{1}} \ldots P_{i_{r}} \in \Delta_{r}$.

Proposition 4.13 Let $r \geq 1$.

(i) We have that

$$
\Delta_{r}=A P_{0}^{r}+\sum_{i=1}^{p} \Delta_{r-1} P_{i} .
$$

(ii) As a left $A$-module $\Delta_{r}$ is spanned by the elements $P_{I}$ where $I$ is admissible of length $r$.

Proof We prove (i) by induction on $r$; it is immediate for $r=1$. Assuming $\Delta_{r}=$ $A P_{0}^{r}+\sum_{i=1}^{p} \Delta_{r-1} P_{i}$, we have that

$$
\begin{array}{rlr}
\Delta_{r+1} & =\Delta_{r} P_{0}+\sum_{j=1}^{p} \Delta_{r} P_{j} \\
& =A P_{0}^{r+1}+\sum_{i=1}^{p} \Delta_{r-1} P_{i} P_{0}+\sum_{j=1}^{p} \Delta_{r} P_{j} \quad \text { by induction } \\
& \subseteq A P_{0}^{r+1}+\sum_{i=1}^{p} \sum_{j=1}^{p} \Delta_{r-1}\left(-x^{i} P_{i}\right) P_{j}+\sum_{j=1}^{p} \Delta_{r} P_{j},
\end{array}
$$

which is contained in $A P_{0}^{r+1}+\sum_{j=1}^{p} \Delta_{r} P_{j}$.

Statement (ii) follows from (i) and induction on $r$. 
Let $\zeta: \Delta \rightarrow \Gamma$ denote the evident map of graded associative rings, induced by sending $\Delta_{1} \subset \Delta$ identically to $\Gamma_{1} \subset \Gamma$; it exists according to the discussion of Section 4.8. We write $\zeta_{r}: \Delta_{r} \rightarrow \Gamma_{r}$ for the restriction of $\zeta$ to the $r$-th grading.

Proposition 4.14 The map $\zeta: \Delta \rightarrow \Gamma$ is an isomorphism.

Proof We show that $\zeta_{r}$ is an isomorphism, by induction on $r$. It is clear that $\zeta_{0}$ and $\zeta_{1}$ are isomorphisms. In the commutative diagram

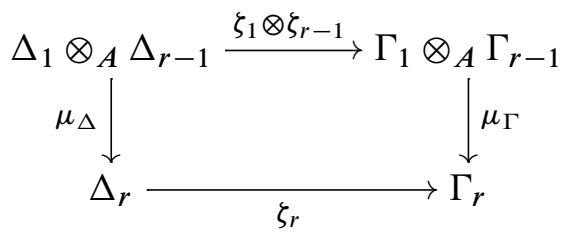

the map $\mu_{\Delta}$ is surjective by construction, $\mu_{\Gamma}$ is surjective by Proposition 4.5 , and $\zeta_{1} \otimes \zeta_{r-1}$ is an isomorphism by induction. Therefore $\zeta_{r}$ is surjective. We also know that $\Gamma_{r}$ is free as a left $A$-module on $1+p+\cdots+p^{r}$ generators, while $\Delta_{r}$ is generated as a left $A$-module by $1+p+\cdots+p^{r}$ elements (the admissible monomials of length $r$ ). Thus $\zeta_{r}$ is an isomorphism.

\subsection{The monomial filtration on $K_{\bullet}^{r}$}

Given sequences $I$ and $J$, we write $I J$ for their concatenation. We define a linear ordering on the set of sequences of length $r$ as follows. We say that $I<J$ if, on writing $I=I^{\prime}(i)$ and $J=J^{\prime}(j)$, we have either (1) $i>j$, or (2) $i=j$ and $I^{\prime}<J^{\prime}$. Thus, sequences of length 1 are ordered:

$$
(p)<(p-1)<\cdots<(1)<(0) .
$$

Sequences of length 2 are ordered:

$(p, p)<\cdots<(0, p)<(p, p-1)<\cdots<(1,1)<(0,1)<(p, 0)<\cdots<(1,0)<(0,0)$.

We write

$$
\Gamma_{r_{1}, \ldots, r_{q}}:=\Gamma_{r_{1}} \otimes_{A} \cdots \otimes_{A} \Gamma_{r_{q}} .
$$

For a sequence $I$ of length $r=\sum_{i=1}^{q} r_{i}$, let $\mathscr{F}_{I} \Gamma_{r_{1}, \ldots, r_{q}}$ denote the left $A$-submodule of $\Gamma_{r_{1}, \ldots, r_{q}}$ spanned by elements of the form $P_{I_{1}} \otimes \cdots \otimes P_{I_{q}}$, where $I_{1} I_{2} \cdots I_{q} \leq I$. Thus we obtain a filtration with $\mathscr{F}_{I} \Gamma_{r_{1}, \ldots, r_{q}} \subseteq \mathscr{F}_{J} \Gamma_{r_{1}, \ldots, r_{q}}$ when $I \leq J$. Observe that $\mathscr{F}_{(0, \ldots, 0)} \Gamma_{r_{1}, \ldots, r_{q}}=\Gamma_{r_{1}, \ldots, r_{q}}$.

We write $\mathscr{F}_{<I} \Gamma_{r_{1}, \ldots, r_{q}}$ for the left $A$-submodule spanned by elements $P_{I_{1}} \otimes \cdots \otimes P_{I_{q}}$ where $I_{1} I_{2} \cdots I_{q}<I$. We write $\operatorname{gr}_{I} \Gamma_{r_{1}, \ldots, r_{q}}=\mathscr{F}_{I} \Gamma_{r_{1}, \ldots, r_{q}} / \mathscr{F}_{<I} \Gamma_{r_{1}, \ldots, r_{q}}$. 
Proposition 4.16 Let $\mu_{i}: \Gamma_{r_{1}, \ldots, r_{q}} \rightarrow \Gamma_{r_{1}, \ldots, r_{i-1}+r_{i}, \ldots, r_{q}}$ be the map induced by multiplication $\Gamma_{r_{i-1}} \otimes_{A} \Gamma_{r_{i}} \rightarrow \Gamma_{r_{i-1}+r_{i}}$. Then for any sequence $I$ of length $r=r_{1}+\cdots+r_{q}$, we have that

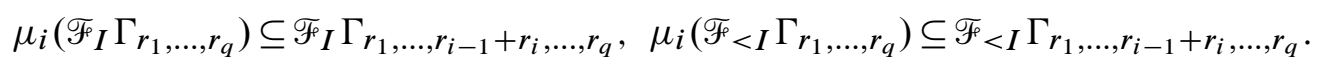

Proof The filtrations are defined as the left $A$-modules spanned by certain monomial elements. The map $\mu_{i}$ is left $A$-linear and preserves the spanning sets.

Warning This filtration does not make $\Gamma$ into a filtered ring. That is, we do not generally have $\mathscr{F}_{I} \Gamma_{r} \cdot \mathscr{F}_{I} I_{r^{\prime}} \subseteq \mathscr{F}_{I} I I^{\prime} \Gamma_{r+r^{\prime}}$, since the subobjects $\mathscr{F}_{I} \Gamma_{r}$ are not right $A$-submodules.

Now we define

$$
\mathscr{F}_{I} K_{q}^{r}:=\bigoplus_{r_{1}+\cdots+r_{q}=r} \mathscr{F}_{I} \Gamma_{r_{1}, \ldots, r_{q}} \subseteq K_{q}^{r} .
$$

The above proposition implies that $\mathscr{F}_{I} K_{\bullet}^{r}$ is a subcomplex of $K_{\bullet}^{r}$. Note further that

$$
\operatorname{gr}_{I} K_{q}^{r}:=\mathscr{F}_{I} K_{q}^{r} / \mathscr{F}_{<I} K_{q}^{r} \approx \bigoplus_{r_{1}+\cdots+r_{q}=r} \operatorname{gr}_{I} \Gamma_{r_{1}, \ldots, r_{q}}
$$

The next proposition shows that the associated gradeds $\operatorname{gr}_{I} K_{q}^{r}$ are free modules, with basis given by elements $P_{I_{1}} \otimes \cdots \otimes P_{I_{q}}$ where $I=I_{1} \cdots I_{q}$ with each $I_{1}, \ldots, I_{q}$ admissible.

Proposition 4.17 Let $I_{1}, \ldots, I_{q}$ be sequences of length $r_{1}, \ldots, r_{q}$ respectively, and let $I=I_{1} \cdots I_{q}$.

(1) If at least one of $I_{1}, \ldots, I_{q}$ is inadmissible, then $\operatorname{gr}_{I} \Gamma_{r_{1} \ldots, r_{q}}=0$.

(2) If all $I_{1}, \ldots, I_{q}$ are admissible, then $\operatorname{gr}_{I} \Gamma_{r_{1}}, \ldots, r_{q}$ is a free left $A$-module on one generator corresponding to $P_{I_{1}} \otimes \cdots \otimes P_{I_{q}}$.

Proof First note that by definition $\operatorname{gr}_{I}=\operatorname{gr}_{I} \Gamma_{r_{1}, \ldots, r_{q}}$ is always a cyclic $A$-module, generated by the image of $P_{I_{1}} \otimes \cdots \otimes P_{I_{q}}$.

We prove (1). Suppose that $I_{k}$ is inadmissible. Then we may write $I_{k}=I_{k}^{\prime}(i, 0) I_{k}^{\prime \prime}$, where $I_{k}^{\prime}$ and $I_{k}^{\prime \prime}$ are two (possibly empty) sequences, and $i \neq 0$. We have that

$$
\begin{aligned}
P_{I_{1}} \otimes \cdots \otimes P_{I_{q}} & =P_{I_{1}} \otimes \cdots \otimes P_{I_{k}^{\prime}} P_{i} P_{0} P_{I_{k}^{\prime \prime}} \otimes \cdots \otimes P_{I_{q}} \\
& =\sum_{j=1}^{p} P_{I_{1}} \otimes \cdots \otimes P_{I_{k}^{\prime}}\left(-x^{j}\right) P_{i} P_{j} P_{I_{k}^{\prime \prime}} \otimes \cdots \otimes P_{I_{q}} .
\end{aligned}
$$


For any $a \in A$, the element $P_{I_{1}} \otimes \cdots \otimes P_{I_{k}^{\prime}} a$ is in $\Gamma_{r_{1}, \ldots, r_{k}^{\prime}}$ (where $r_{k}^{\prime}$ is the length of $I_{k}^{\prime}$ ), and so is a left $A$-linear combination of monomials of the form $P_{J_{1}} \otimes \cdots \otimes P_{J_{k}}$. Thus, $P_{I_{1}} \otimes \cdots \otimes P_{I_{q}}$ is a left $A$-linear combination of monomials of the form $P_{J_{1}} \otimes \cdots P_{J_{k}} P_{i} P_{j} P_{I_{k}^{\prime \prime}} \otimes \cdots \otimes P_{I_{q}}$ with $j \neq 0$. Since

$$
J_{1} \cdots J_{k}(i, j) I_{k}^{\prime \prime} \cdots I_{q}<I_{1} \cdots I_{k^{\prime}}(i, 0) I_{k^{\prime \prime}} \cdots I_{q},
$$

it follows that $\operatorname{gr}_{I} \Gamma_{r_{1}, \ldots, r_{q}}=0$, proving (1).

To prove (2), observe that from (1) we may conclude that $\Gamma_{r_{1}, \ldots, r_{q}}$ is spanned as a left $A$-module by elements of the form $P_{I_{1}} \otimes \cdots \otimes P_{I_{q}}$ with $I_{1}, \ldots, I_{q}$ admissible. Since $\Gamma_{r_{1}, \ldots, r_{q}}$ is a free left $A$-module, with rank equal to the number of such collections of admissible sequences, the result follows.

Given an abstract simplicial complex $X$ with some chosen ordering of its vertices, let $C_{\bullet}(X)$ denote the chain complex associated to $X$, and let $\widetilde{C}_{\bullet}(X)$ denote the mapping fiber of the augmentation map $C_{\bullet}(X) \rightarrow \mathbb{Z}$, where $\mathbb{Z}$ is viewed as a chain complex concentrated in degree 0 . Thus, $\widetilde{C}_{q}(X)$ is the free abelian group on the $q$-simplices of $X$ for $q \geq 0$, and $\widetilde{C}_{-1}(X)=\mathbb{Z}$.

Let $\Delta^{n}$ denote the $n$-simplex viewed as a simplicial complex. The vertices of $\Delta^{n}$ are elements of $S=\{1, \ldots, n+1\}$, and a $q$-simplex of $\Delta^{n}$ is a subset of size $q+1$ of $S$. Observe that $\Delta^{-1}$ is a simplicial complex whose realization is the empty space.

The following is elementary and standard; it amounts to the fact that the quotient $\left|\Delta^{n}\right| /|Y|$ of the $n$-simplex by a subcomplex which is a union of codimension 1 faces is either contractible or homeomorphic to a sphere.

Proposition 4.18 If $\Delta^{n}$ is the $n$-simplex viewed as a simplicial complex, and if $Y_{1}, \ldots, Y_{d} \subset \Delta^{n}$ is a (possibly empty) collection of distinct codimension 1 faces of $\Delta^{n}$, then

$$
H_{q}\left[\tilde{C}_{\bullet}\left(\Delta^{n}\right) / \sum_{i=1}^{d} \tilde{C}_{\bullet}\left(Y_{i}\right)\right]=0 \quad \text { if } q \neq n \text { or } d<n+1 .
$$

If $q=n$ and $d=n+1$, then $H_{n}\left(\tilde{C}_{\bullet}\left(\Delta^{n}\right) / \sum \tilde{C}_{\bullet}\left(Y_{i}\right)\right)=\mathbb{Z}$.

Proposition 4.19 Let $I=\left(i_{1}, \ldots, i_{r}\right)$ be a sequence. Then there is an isomorphism of chain complexes

$$
\operatorname{gr}_{I} K_{\bullet}^{r} \approx\left[\tilde{C}_{\bullet-2}\left(\Delta^{r-2}\right) / \sum_{i=1}^{d} \tilde{C}_{\bullet-2}\left(Y_{i}\right)\right] \otimes_{\mathbb{Z}} A,
$$


where $Y_{1}, \ldots, Y_{d}$ is a collection of distinct codimension 1 faces of $\Delta^{r-2}$. Here $d$ is size of the set

$$
T=\left\{k \in\{1, \ldots, r-1\} \mid i_{k} \neq 0 \text { and } i_{k+1}=0\right\} .
$$

Thus, $H_{q} \operatorname{gr}_{I} K_{\bullet}^{r}=0$ unless $q=r$, and $H_{r} \operatorname{gr}_{I} K_{\bullet}^{r}$ is a free $A$-module (of rank 0 or 1 , depending on $I$ ).

Proof Given a sequence $I=\left(i_{1}, \ldots, i_{r}\right)$, we define maps

$$
\phi_{I}: \tilde{C}_{q-2}\left(\Delta^{r-2}\right) \rightarrow K_{q}^{r}=\bigoplus_{r_{1}+\cdots+r_{q}=r} \Gamma_{r_{1}, \ldots, r_{q}}
$$

as follows. If $s=\left[1 \leq s_{1}<\cdots<s_{q-1} \leq r-1\right]$ is a $(q-2)-$ simplex in $\Delta^{r-2}$, then let $\phi_{I}(s)=P_{I_{1}} \otimes \cdots \otimes P_{I_{q}}$, where

$$
I_{k}=\left(i_{s_{k-1}+1}, \ldots, i_{s_{k}}\right) \quad \text { for } k=1, \ldots, q \text {, taking } s_{0}=0 \text { and } s_{q}=r .
$$

Note that $I=I_{1} \cdots I_{q}$.

Thus $\phi_{I}: \widetilde{C}_{\bullet-1}\left(\Delta^{r-2}\right) \rightarrow K_{\bullet}^{r}$ is a chain map, and in fact the image of $\phi_{I}$ is contained in $\mathscr{F}_{I} K_{\bullet}^{r}$.

Given $k \in\{1, \ldots, r-1\}$, let $Y_{k} \subset \Delta^{r-2}$ denote the subcomplex consisting of the codimension 1 face spanned by all vertices except $k$. Let $Y_{k_{1}}, \ldots, Y_{k_{d}}$ be the collection of all such faces for which $i_{k_{j}} \neq 0$ and $i_{k_{j}+1}=0$. By Proposition 4.17 (1) it follows that $\phi_{I}$ factors through a map

$$
\tilde{C}_{\bullet-2}\left(\Delta^{r-2}\right) / \sum_{j=1}^{d} \tilde{C}_{\bullet-2}\left(Y_{k_{j}}\right) \rightarrow \mathscr{F}_{I} K_{\bullet}^{r} / \mathscr{F}_{<I} K_{\bullet}^{r} .
$$

By Proposition 4.17 (2), we see that this passes to an isomorphism

$$
A \otimes_{\mathbb{Z}} \widetilde{C}_{\bullet-2}\left(\Delta^{r-2}\right) / \sum \widetilde{C}_{\bullet-2}\left(Y_{k_{j}}\right) \approx \operatorname{gr}_{I} K_{\bullet}^{r} .
$$

Proposition 4.20 For all $r \geq 0$, we have that $H_{j} K_{\bullet}^{r}=0$ if $j \neq r$, and that $H_{r} K_{\bullet}^{r}$ is a finitely generated free $A$-module.

Proof The spectral sequence $E_{1}^{q, I}=H_{q} \operatorname{gr}_{I} K_{\bullet}^{r} \Longrightarrow H_{*} K_{\bullet}^{r}$ collapses trivially, since $E_{1}^{q, I}=0$ unless $q=r$. 


\section{Appendix: The polynomials $F_{m}(x, y)$}

For $m \in \mathbb{N}$ let $F_{m}(x, y) \in \mathbb{Z}[x, y]$ denote the polynomial

$$
F_{m}(x, y)=\prod_{m=d e}\left(x^{d}-y^{e}\right)
$$

where $d, e$ range over all pairs of natural numbers such that $m=d e$.

Let $R$ be a commutative ring. We propose to define a category $D(R)$ as follows. The objects of $D(R)$ are the elements of the ring $R$. The morphisms are given by

$$
\operatorname{Hom}_{D(R)}(a, b)=\left\{m \in \mathbb{N} \mid F_{m}(a, b)=0\right\} .
$$

We write $\langle m\rangle: a \rightarrow b$ for the morphism corresponding to $m \in \mathbb{N}$. Identity morphisms are those of the form $\langle 1\rangle: a \rightarrow a$.

We define the composition of $\langle m\rangle: a \rightarrow b$ with $\langle n\rangle: b \rightarrow c$ to be $\langle m n\rangle: a \rightarrow c$. It is clear that this will make $D(R)$ into a category, as long as composition is well-defined. That is, $D(R)$ is a category if $F_{m}(a, b)=0$ and $F_{n}(b, c)=0$ implies $F_{m n}(a, c)=0$ for all $a, b, c \in R$ and $m, n \in \mathbb{N}$.

We will show that with this composition law, $D(R)$ is in fact a category for every $R$. Equivalently, we show that for all $m, n$, the polynomial $F_{m n}(x, z)$ is contained in the ideal $\left(F_{m}(x, y), F_{n}(y, z)\right)$ of $\mathbb{Z}[x, y, z]$.

Lemma 4.21 If $R$ is an integral domain, then $D(R)$ is a category. Thus, for every $m, n$, there exists an $N \geq 1$ such that $F_{m n}(x, z)^{N} \in\left(F_{m}(x, y), F_{n}(y, z)\right)$.

Proof If $a, b, c \in R$ satisfy $F_{m}(a, b)=0=F_{n}(b, c)$, then since $R$ is a domain there must exist $d, e, d^{\prime}, e^{\prime} \in \mathbb{N}$ with $m=d e, m^{\prime}=d^{\prime} e^{\prime}$, such that $a^{d}=b^{e}$ and $b^{d^{\prime}}=c^{e^{\prime}}$, whence $a^{d d^{\prime}}=b^{d^{\prime} e}=c^{e e^{\prime}}$, whence $F_{m n}(a, c)=0$.

Let $T_{m}(x, y)=\mathbb{Z}[x, y] /\left(F_{m}(x, y)\right)$.

Lemma 4.22 Let $K$ be a field of characteristic 0 , and let $\phi: \mathbb{Z}[x] \rightarrow K$ be a ring homomorphism such that $a=\phi(x)$ is neither 0 nor a root of unity. Then $A=$ $K \otimes_{\mathbb{Z}[x]} T_{m}(x, y)$ is isomorphic to a finite product $\prod K_{i}$ of fields. Furthermore, for each $i$ the evident homomorphism $T_{m}(x, y) \rightarrow A \rightarrow K_{i}$ sends $y$ to an element $b_{i} \in K_{i}$ which is neither 0 nor a root of unity. 
Proof We have that $A \approx K[y] /\left(T_{m}(a, y)\right)$. To show that $A$ is a product of fields, it suffices to show that the polynomial $T_{m}(a, y) \in K[y]$ has no repeated roots in the algebraic closure $\bar{K}$ of $K$. The polynomial $T_{m}(a, y)$ is a product (up to sign) of factors of the form $g_{d}(y)=y^{d}-a^{e}$ where $m=d e$. It is clear that each $g_{d}$ has $d$ distinct roots, of the form $\zeta \sqrt[d]{a^{e}}$ where $\zeta \in \mu_{d}(\bar{K})$ and $\sqrt[d]{a^{e}}$ some chosen $d$-th root of $a^{e}$. If $\beta \in \bar{K}$ such that $g_{d}(\beta)=0=g_{d^{\prime}}(\beta)$ where $m=d e=d^{\prime} e^{\prime}$ with $e>e^{\prime}$, it is straightforward to show that $a^{e^{2}}=\beta^{m}=a^{e^{\prime 2}}$, whence $a^{e^{\prime 2}}\left(a^{e^{2}-e^{\prime 2}}-1\right)=0$, which is impossible by the hypothesis on $a$. Thus no roots of $T_{m}(a, y)$ are repeated.

The homomorphism $T_{m}(x, y) \rightarrow K_{i}$ sends $y$ to an element $b_{i}$ with the property that $b_{i}^{d}=a^{e}$ for some $m=d e$. Since $a$ is not 0 or a root of unity, neither is $b_{i}$.

Proposition 4.23 For all $m, n \geq 1$, the polynomial $F_{m n}(x, z)$ is an element of the ideal $\left(F_{m}(x, y), F_{n}(y, z)\right)$ of $\mathbb{Z}[x, y, z]$. Thus, for every commutative ring $R, D(R)$ is a well-defined category.

Proof It suffices to show that the ring

$$
T_{m}(x, y) \otimes_{\mathbb{Z}[y]} T_{n}(y, z) \approx \mathbb{Z}[x, y, z] /\left(F_{m}(x, y), F_{n}(y, z)\right)
$$

has no nilpotents; since we have already shown that $F_{m n}(x, y)$ is nilpotent in this ring, we will thus have $F_{m, n}(x, y) \in\left(F_{m}(x, y), F_{n}(y, z)\right)$.

Let $K=\mathbb{Q}(x)$, viewed as a $\mathbb{Z}[x]$-algebra. The elements $F_{m}(x, y)$ are monic as polynomials in $y$ with coefficients in $\mathbb{Z}[x]$ (up to sign); thus the maps $T_{m}(x, y) \rightarrow$ $K \otimes_{\mathbb{Z}[x]} T_{m}(x, y)$ and $T_{m}(x, y) \otimes_{\mathbb{Z}[y]} T_{n}(y, z) \rightarrow K \otimes_{\mathbb{Z}[x]} \otimes T_{m}(x, y) \otimes_{\mathbb{Z}[y]} T_{n}(y, z)$ are monomorphisms. Hence, it suffices to show that $K \otimes_{\mathbb{Z}[x]} T_{m}(x, y) \otimes_{\mathbb{Z}[y]} T_{n}(y, z)$ has no nilpotents.

By Lemma 4.22, we see that $K \otimes_{\mathbb{Z}[x]} T_{m}(x, y) \approx \prod_{i} K_{i}$ where $K_{i}$ are fields. A second application of the lemma shows that $K_{i} \otimes_{\mathbb{Z}[y]} T_{n}(y, z) \approx \prod_{j} K_{i j}$ where $K_{i j}$ are fields, whence $K \otimes_{\mathbb{Z}[x]} T_{m}(x, y) \otimes_{\mathbb{Z}[y]} T_{n}(y, z) \approx \prod_{i, j} K_{i j}$, which clearly has no nilpotents.

\section{References}

[1] M H Baker, E González-Jiménez, J González, B Poonen, Finiteness results for modular curves of genus at least 2, Amer. J. Math. 127 (2005) 1325-1387 MR2183527

[2] M Behrens, T Lawson, Isogenies of elliptic curves and the Morava stabilizer group, J. Pure Appl. Algebra 207 (2006) 37-49 MR2244259

[3] T Kashiwabara, K(2)-homology of some infinite loop spaces, Math. Z. 218 (1995) 503-518 MR1326982 
[4] N M Katz, B Mazur, Arithmetic moduli of elliptic curves, Annals of Math. Studies 108, Princeton Univ. Press (1985) MR772569

[5] B Poonen (mathoverflownet/users/2757), Supersingular elliptic curves and their "functorial" structure over $F_{p}^{2}$, MathOverflow (2010) Available at http:// mathoverflow.net/questions/19013

[6] S B Priddy, Koszul resolutions, Trans. Amer. Math. Soc. 152 (1970) 39-60 MR0265437

[7] D Quillen, Finite generation of the groups $K^{i}$ of rings of algebraic integers, from: "Algebraic $K$-theory, I: Higher $K$-theories (Proc. Conf., Battelle Memorial Inst., Seattle, WA, 1972)", (H Bass, editor), Lecture Notes in Math. 341, Springer, Berlin (1973) 179-198 MR0349812

[8] C Rezk, Rings of power operations for Morava E-theories are Koszul arXiv: 1204.4831

[9] C Rezk, The congruence criterion for power operations in Morava E-theory, Homology, Homotopy Appl. 11 (2009) 327-379 MR2591924

[10] L Solomon, The Steinberg character of a finite group with BN-pair, from: "Theory of Finite Groups (Symposium, Harvard, Cambridge, MA, 1968)”, Benjamin, New York (1969) 213-221 MR0246951

[11] N P Strickland, Finite subgroups of formal groups, J. Pure Appl. Algebra 121 (1997) 161-208 MR1473889

[12] N P Strickland, Morava E-theory of symmetric groups, Topology 37 (1998) 757-779 MR1607736

Department of Mathematics, University of Illinois at Urbana-Champaign 273 Altgeld Hall, MC-382, 1409 W Green Street, Urbana IL 61801, United States rezk@math.uiuc.edu

http://www. math.uiuc.edu/ rezk

Received: 21 March $2011 \quad$ Revised: 18 March 2012 